\title{
ATOMIC THEORY METHODS FOR THE POLARIZATION IN PHOTON AND ELECTRON INTERACTIONS WITH ATOMS
}

\author{
A. Kupliauskienè \\ Vilnius University Research Institute of Theoretical Physics and Astronomy, A. Goštauto 12, LT-01108 Vilnius, Lithuania \\ E-mail: akupl@itpa.lt
}

Received 20 February 2004

Dedicated to the 100th anniversary of Professor A. Jucys

\begin{abstract}
An alternative method to the density matrix formalism for the derivation of general expressions for the cross-sections of the interaction of polarized atoms with polarized photons and electrons is presented. The expression for the cross-section describing the polarization states of all particles taking part in the process are obtained in the form of the expansion via irreducible tensors that have the simplest possible behaviour under changes of directions. The ways of the application of the general expressions suitable for the specific experimental conditions are outlined by deriving asymmetry parameters of the angular distributions of photoelectrons and Auger electrons following photoionization as well as the parameters of the angular correlations between photo- and Auger electrons.
\end{abstract}

Keywords: photon interactions with atoms, electron scattering

PACS: $32.80 .-\mathrm{t}, 34.80 .-\mathrm{i}$

\section{Introduction}

In collision processes, the differential cross-section is simply a scalar with respect to the joint rotation of incoming and outgoing particles if neither the projection quantum numbers of the incident particle nor the target are resolved. For the derivation of expressions for the spectroscopic characteristics invariant under space rotation, the powerful methods of atomic theory and angular momentum were developed by A. Jucys and his coworkers $[1,2]$ in the case of the states of atoms described with complex configurations. These methods can also be applied for the study of the interaction of atoms with photons, electrons, and other charged particles that is a powerful tool for the investigation of matter and interactions and have both theoretical and practical importance. In any atomic process, but particularly in collisions, energy, momentum, and angular momentum are exchanged among the various constituents. All three of these quantities are conserved, and, in a classical theory, all three quantities may simultaneously have fixed values. Quantum states, however, cannot be simultaneously eigenstates of linear and angular momentum. If the state of linear momentum is fixed, as it usually is in collision experiments, then the angular momentum is not. Angular momentum is conserved, but the information about it cannot be used directly. The mean value of products of the components of angular momentum that are proportional to the parameters describing the orientation and alignment can be described. Just these products completely specify atomic states [3]. The measurement of the parameters of the alignment and orientation helps us to learn about the interchange of angular momentum in atomic collisions. Orientation and alignment parameters essentially characterize, respectively, the circulation of the atomic electron around the atomic core and the shape of the excited electron cloud and its direction in space. Orientation and alignment parameters thus allow us to go beyond the cross-section concept, in favourable cases leading to the so-called perfect scattering experiment, in which the sets of quantum-mechanical scattering amplitudes and phases are completely determined [4].

The studies of the polarization phenomena in the interactions of atoms with charged particles and radiation stimulated the creation of new methods of the investigation of plasmas, ionized gases [5], and solids [6]. One of them is plasma polarization spectroscopy [5], since in the majority of laboratory and astrophysical plasmas the electron-ion interaction is the dominant mechanism of the radiation emission. Measurements 
of the polarization of the spectroscopic characteristics of plasma provide a unique possibility for the diagnostics of electron and ion distribution function with high accuracy. The distortion of the Maxwellian distribution function or presence in plasma beams may play a substantial role for the formation of the emission spectra [6]. The deviations from the Maxwellian distribution of electrons were directly registered in laser produced [7], tokamak [8], vacuum spark [9], and astrophysical (Solar corona) [10] plasmas by observing the polarization of line spectra and continuous radiation. Polarization state of emission and absorption lines could be considered as a consequence of polarization of atoms due to nonequilibrium populations of magnetic sublevels or to ordering of angular momenta of atomic particles in plasma, that is called a self-alignment phenomenon caused by the anisotropy properties of plasma sources.

Disentanglement of the geometrical aspects of anisotropy from the dynamical ones is another aspect of alignment and orientation. From the classical radiation theory, it follows that when the light is observed, only the components of the source projected onto a plane perpendicular to the direction of observation are imaged. The source must be looked at from different angles to determine its complete electromagnetic configuration. This holds for any measurement of radiation. To see the complete source, one must look at it from several angles. Irreducible tensors are selected for the description of polarization precisely because they have the simplest possible behaviour under changes of direction (rotation) [3].

The experiments on free polarized atoms open up the possibility to disentangle the atomic and solid state effects [11]. Because of some atoms [11,12] basic practical importance for the magnetic properties of multilayer systems and of ultrathin films on ferromagnetic substrates they are currently the focus of many investigations. The photoelectron spectroscopy, exploring linear and circular magnetic dichroism [13] yields detailed and site-specific information [11,12]. Magnetooptical effects in the VUV and soft X-ray ranges are very important tools for the investigation of magnetic materials [13].

To derive expressions for the parameters suitable to characterize the polarization state in the photoionization of atoms, the density matrix formalism proposed by Fano and Macek $[14,15]$ has been widely used [16-23]. In this formalism the polarization state of atoms or ions was described by the statistical tensors (state multipoles) which were the basis for the expansion of atomic density matrix [24]. The methods of the density matrix became common, but the work by Fano and Macek [15] formulated a transparent method for the light emission in the decay of a stationary state of an atom following the excitation by collision with an electron or photon that used a complete set of mean values of measurable quantities instead of the density matrix to characterize a state, thereby bypassing the language of the density matrix [3]. The Wigner-Eckart theorem [1] was used to relate measured quantities to mean values of irreducible tensors constructed from angular momentum operators. In effect, these mean values were proportional to state multipoles, therefore, density matrix elements need never appear. Then the matrix element is expressed as a product of the reduced matrix element invariant under space rotation and the Clebsch-Gordan coefficient depending on the orientation in space.

A further development of the ideas of Fano and Macek [15] was accomplished by Kupliauskienè et al. $[25,26]$. They have used the method based on the atomic theory $[1,2,27]$ to derive the expressions for the photoionization cross-sections of polarized atoms by polarized radiation. The methods of the theory of an atom $[1,2,27]$ usually were used to the isolated atom for the derivation of the expressions for the spectroscopic characteristics that are invariant under the rotation of the space. These characteristics were made independent of the magnetic components of the total angular momentum by using the Wigner-Eckart theorem [1]. Then, the matrix element was expressed as a product of the reduced matrix element that was invariant under space rotation and the Clebsch-Gordan coefficient depending on the orientation in space. But the Clebsch-Gordan coefficients in the expression for the probability or cross-section can also be used to construct the sums of the spherical tensors for the description of the orientation in space and rotation properties. Until the Vilnius theoreticians have started the application of the atomic theory methods for the investigation of the polarization in atoms $[25,26]$ there have been few applications of this method, and these have been restricted to the polarization parameters for special cases. Fano and Dill [28] have obtained the expression for the photoelectron angular distribution parameter $\beta$ expressed as a sum of incoherent contributions corresponding to the different magnitudes of the angular momentum transferred to an unpolarized target. The same method was extended by Klar [18] to the angular distribution of spin-polarized photoelectrons from unpolarized atoms. The expression for the angular distribution of Auger electrons following ionization by a 
beam of unpolarized electrons or protons was also derived without the help of density matrix by Cleff and Mehlhorn [29]. Therefore, the formulation of the polarization theory of atoms in general form based on the traditional technique of spherical tensors and wave functions is useful for people working in atomic theory.

The aim of the method based on the atomic theory approach was the derivation of the differential crosssection and parameters describing the interaction of polarized atoms with polarized radiation, electrons or other charged particles in the form of the multiple expansions over spherical tensors by using the graphical technique [30-35] for the integration over the angular and spin variables of the matrix elements of the transition operators. Any calculation made using the graphical technique can also be made using conventional algebraic technique [31]. To every graphical reduction there is a corresponding algebraic reduction because of the correspondence between graphs and algebraic formulas. However, the graphical method has two advantages over the algebraic methods: (a) the notation is more compact because the undetermined magnetic quantum numbers need not be written explicitly, and (b) reductions can be made by recognizing geometrical patterns. The graphical technique of angular momentum was proposed by Levinson [30] to obtain an expression for the reduced matrix element invariant under the rotation of space. The dependence of the matrix element on the magnetic quantum numbers was separated with the help of Wigner-Eckart theorem. In [25, 26], the graphical technique was extended to make it suitable for investigating the probability of the process depending on the mutual orientation of the particles participating in the process or on their orientation with respect to the chosen quantization axis.

The present work is devoted to the review of the applications of the alternative method to the density matrix formalism for the investigation of the excitation and ionization of polarized atoms and ions by polarized radiation, electrons, and other charged particles with subsequent decay of the formed ions and atoms in two-step approximation. The general expressions for the cross-sections of the excitation of atoms by electrons as well as the radiative and dielectronic recombinations are derived and presented for the first time. The expression for the photoionization cross-section is also written in a more general form more convenient for the practical applications. The photon-atom interaction will be described in Section 2 where the excitation and ionization of polarized atoms by polarized photons as well as the modifications of the expressions for the probabilities or cross-sections in one- and multistep approximation will be discussed. In Section 3, the electron-atom interactions (excitation and ionization of atoms and ions and radiative recombination) are considered. The radiative and Auger decay processes are described in Section 4. The two-step process - dielectronic recombination - is investigated in Section 5. Section 6 is devoted to demonstrate some applications of the general expressions for the specific experimental conditions. The review ends with concluding remarks that summarize the results and discuss the possibilities of the application of ordinary atomic theory for the description of the polarization in photon-atom and electron-atom interactions.

\section{Photon-atom interactions}

\subsection{Photoexcitation of atoms}

The laser [36] and tunable synchrotron radiation allows one not only to ionize an electron of a specific outer or inner shell of an atom but also to excite it to a specific orbital $[37,38]$. The state of the produced atom is polarized if the radiation is polarized. Thus, the excitation of atoms by polarized radiation is one of the ways to produce atoms in polarized states for further measurements $[39,40]$. The photoexcitation can be used as a first step to create a resonant state with well-defined angular momentum and parity for further investigations of polarization and angular correlation phenomena $[41,23]$.

The general expression for the excitation of polarized atoms by polarized radiation was obtained [42] for the following process:

$$
A\left(\alpha_{0} J_{0} M_{0}\right)+h \nu\left(\widehat{\epsilon}_{\lambda} \mathbf{k}_{0}\right) \rightarrow A^{*}\left(\alpha_{1} J_{1} M_{1}\right) .
$$

Here an atom $A$ in the state $\alpha_{0} J_{0} M_{0}$ is excited by the electromagnetic radiation into the state $\alpha_{1} J_{1} M_{1}$, $\alpha_{0}$ and $\alpha_{1}$ define the configuration and other quantum numbers, $J$ is the total angular momentum and $M$ is its projection. The electromagnetic radiation is described with the wave vector $\mathbf{k}_{0}\left(k_{0}=\left|\mathbf{k}_{0}\right|=\omega / c\right.$, $\omega=2 \pi \nu$, and $\nu$ is the frequency of light) and unit vector $\widehat{\epsilon}_{\lambda}$ of the polarization ( $\lambda$ is the helicity, $\lambda= \pm 1$ ). The system of atomic units is used in the present work ( $\hbar=e=m=1, c=137$ unless these constants are displayed explicitly). The assumption is taken into account that the fine structure splitting $\gg$ line width $\gg$ hyperfine structure splitting. Then the states of an atom can be specified by the total angular momentum $J$ of all electronic shells. The modifications enabling the 
calculations of the probability in the case when hyperfine structure is important will be described below. In the present work, it is assumed that the directions for the measurement of the projections $M_{0}$ and $M_{1}$ can be different.

The differential cross-section of the process (1) can be written as follows [23]:

$$
\begin{aligned}
& \frac{\mathrm{d} \sigma\left(\alpha_{0} J_{0} M_{0} \widehat{\epsilon}_{\lambda} \mathbf{k}_{0} \rightarrow \alpha_{1} J_{1} M_{1}\right)}{\mathrm{d} \Omega} \\
& =2 \pi^{2}\left[\int\left\langle\alpha_{1} J_{1} M_{1}|\mathbf{J}(\mathbf{r})| \alpha_{0} J_{0} M_{0}\right\rangle \mathbf{A}_{\lambda \mathbf{k}_{0}}(\mathbf{r}) \mathrm{d} \mathbf{r}\right] \\
& \quad \times\left[\int\left\langle\alpha_{1} J_{1} M_{1}|\mathbf{J}(\mathbf{r})| \alpha_{0} J_{0} M_{0}\right\rangle \mathbf{A}_{\lambda \mathbf{k}_{0}}(\mathbf{r}) \mathrm{d} \mathbf{r}\right]^{*} .
\end{aligned}
$$

Here $\mathbf{J}(\mathbf{r})$ stands for the operator of the current of electrons and $\mathbf{A}_{\lambda \mathbf{k}_{0}}(\mathbf{r})$ is the operator of the vector potential of electromagnetic field. Taking into account that $k r \gg 1$ and the case of an arbitrary direction of the incidence $\mathbf{k}_{0}$ of the photon, and inserting the multipole expansion for the $\mathbf{A}_{\lambda \mathbf{k}_{0}}(\mathbf{r})$, the expression in the right brackets of Eq. (2) acquires the form [23]

$$
\begin{aligned}
\int\left\langle\alpha_{1} J_{1} M_{1}|\mathbf{J}(\mathbf{r})| \alpha_{0} J_{0} M_{0}\right\rangle \mathbf{A}_{\lambda k_{0}}(\mathbf{r}) \mathrm{d} \mathbf{r} \\
=\sum_{p=0,1} \sum_{k=1}^{\infty} \sum_{q=-k}^{q=k}\left\{\mathrm{i}^{k}(-\mathrm{i} \lambda)^{p}\left[\frac{k+1}{k}\right]^{1 / 2} \frac{k_{0}^{k-1 / 2}}{(2 k-1) ! !}\right. \\
\left.\quad \times D_{q \lambda}^{k}\left(\widehat{k}_{0}\right)\left\langle\alpha_{1} J_{1} M_{1}\left|\mathcal{Q}_{k q}^{p}\right| \alpha_{0} J_{0} M_{0}\right\rangle\right\} \\
=\sum_{k=1}^{\infty} \sum_{q=-k}^{q=k}\left\langle\alpha_{1} J_{1} M_{1}\left|Q_{q}^{(k)}\right| \alpha_{0} J_{0} M_{0}\right\rangle D_{q \lambda}^{k}\left(\widehat{k}_{0}\right), \quad(3) \\
\left\langle\alpha_{1} J_{1} M_{1}\left|Q_{q}^{(k)}\right| \alpha_{0} J_{0} M_{0}\right\rangle \\
=k_{0}^{k-1 / 2} \sum_{p=0,1}\left[\frac{k+1}{k}\right]^{1 / 2} \frac{\mathrm{i}^{k}(-\mathrm{i} \lambda)^{p}}{(2 k-1) ! !} \\
\quad \times\left\langle\alpha_{1} J_{1} M_{1}\left|\mathcal{Q}_{k q}^{p}\right| \alpha_{0} J_{0} M_{0}\right\rangle .
\end{aligned}
$$

Here $D_{q \lambda}^{k}\left(\widehat{k}_{0}\right)$ is the Wigner rotation matrix [43] for transforming from the helicity frame $\left(\widehat{k}_{0}\right.$ is the direction of the incoming radiation) to the frame common to all particles participating in the process and used for the evaluation of reduced matrix elements, $p=0$ in- dicates the operator of the electric multipole transition (Ek) [27]

$$
\mathcal{Q}_{k q}^{0}=-r^{k} C_{q}^{(k)},
$$

and $p=1$ shows the operator of the magnetic multipole transition $(\mathrm{M} k)$

$$
\begin{aligned}
\mathcal{Q}_{k q}^{1}= & -\frac{1}{c}[k(2 k-1)]^{1 / 2} r^{k-1} \\
& \times\left\{\frac{1}{k+1}\left[C^{(k-1)} \times L^{(1)}\right]_{q}^{(k)}\right. \\
& \left.+\left[C^{(k-1)} \times S^{(1)}\right]_{q}^{(k)}\right\} .
\end{aligned}
$$

Here $L^{(1)}$ and $S^{(1)}$ are the operators of the orbital and spin angular momentum, respectively, $C_{q}^{(k)}$ is the operator of the spherical function normalized to $[4 \pi /(2 k+1)]^{1 / 2}[1]$.

Note that the parities of the magnetic and electric multipole fields are $(-1)^{k}$ and $(-1)^{k+1}$, respectively. Only the magnetic $(\mathrm{M} k)$ and electric $(\mathrm{E} k)$ part contributes between specific electronic states owing to parity selection rules. Since we are considering pure photon states, there is no need to introduce Stokes parameters explicitly. In electrical dipole approximation, the matrix element (4) is as follows:

$$
\begin{aligned}
& \left\langle\alpha_{1} J_{1} M_{1}\left|Q_{q}^{(1)}\right| \alpha_{0} J_{0} M_{0}\right\rangle \\
& \quad=\sqrt{2 k_{0}}\left\langle\alpha_{1} J_{1} M_{1}\left|\mathcal{Q}_{1 q}^{0}\right| \alpha_{0} J_{0} M_{0}\right\rangle .
\end{aligned}
$$

The helicity $\lambda= \pm 1$ describes the right-hand and left-hand circular polarization of the radiation. In the case of any polarization $\widehat{\epsilon}$, the polarization of radiation may be expressed via the circular polarization. Then

$$
\mathbf{A}_{\epsilon \mathbf{k}_{0}}(\mathbf{r})=\alpha \mathbf{A}_{\lambda=+1 \mathbf{k}_{0}}(\mathbf{r})+\beta \mathbf{A}_{\lambda=-1 \mathbf{k}_{0}}(\mathbf{r})
$$

that has to be inserted into Eq. (3) to obtain the expression for the excitation cross-section (2).

Sometimes it is more convenient to analyse the polarization state of the particle with respect to the direction that differs from the one used for the calculation of the matrix element where all particles of the process (1) should be described in the same coordinate system and their projections of the angular momentum on the same quantization axis. For the transfer from the wave function $|J M\rangle$ defined in the laboratory fixed direction to the wave function $|J \widetilde{M}\rangle$ of the atomic frame used for the evaluation of reduced matrix elements, the coordinate rotation transformation [43] is used: 


$$
|J M\rangle=\sum_{\widetilde{M}} D_{\widetilde{M} M}^{J}(\widehat{J})|J \widetilde{M}\rangle
$$

Here $D_{\widetilde{M} M}^{J}(\widehat{J})$ denotes the Wigner rotation matrix [43], and the hat on $J$ indicates the rotation by solid angle that transforms the atomic frame into the laboratory one used for the polarization measurements of the characteristics depending on $\mathbf{J}$. In the laboratory system of coordinates, the matrix element $\left\langle\alpha_{1} J_{1} M_{1}\left|Q_{q}^{(k)}\right| \alpha_{0} J_{0} M_{0}\right\rangle$ can be written by taking into account Eq. (9) in the form

$$
\left\langle\alpha_{1} J_{1} M_{1}\left|Q_{q}^{(k)}\right| \alpha_{0} J_{0} M_{0}\right\rangle=\sum_{\widetilde{M}_{0}, \widetilde{q}, \widetilde{M}_{1}}\left\langle\alpha_{1} J_{1} \widetilde{M}_{1}\left|Q_{\widetilde{q}}^{(k)}\right| \alpha_{0} J_{0} \widetilde{M}_{0}\right\rangle D_{\widetilde{M}_{0} M_{0}}^{J_{0}}\left(\widehat{J}_{1}\right) D_{\widetilde{q} q}^{k}\left(\widehat{k}_{0}\right) D_{\widetilde{M}_{1} M_{1}}^{* J_{1}}\left(\widehat{J}_{1}\right) .
$$

The matrix element $\left\langle\alpha_{1} J_{1} \widetilde{M}_{1}\left|Q_{\widetilde{q}}^{(k)}\right| \alpha_{0} J_{0} \widetilde{M}_{0}\right\rangle$ is defined in the atomic frame. The angular momentum part of this matrix element was obtained in [42] with the help of the graphical technique of the angular momentum [1]. The final expression for the excitation cross-sections is

$$
\begin{aligned}
& \frac{\mathrm{d} \sigma\left(\alpha_{0} J_{0} M_{0} \widehat{\epsilon}_{q} \mathbf{k}_{0} \rightarrow \alpha_{1} J_{1} M_{1}\right)}{\mathrm{d} \Omega} \\
& =C \sum_{K_{0}, K_{r}, K_{1}, k, k^{\prime}} \frac{1}{2 K_{1}+1} \mathcal{A}^{r}\left(K_{0}, K_{r}, K_{1}, k, k^{\prime}\right) \sum_{N_{0}, N_{r}, N_{1}, N_{1}^{\prime}}\left[\begin{array}{c}
K_{0} K_{r} K_{1} \\
N_{0} N_{r} N_{1}
\end{array}\right] T_{N_{0}}^{* K_{0}}\left(J_{0}, J_{0}, M_{0} \mid \widehat{J}_{0}\right) \\
& \quad \times T_{N_{r}}^{* K_{r}}\left(k, k^{\prime}, q \mid \widehat{k}_{0}\right) T_{N_{1} N_{1}^{\prime}}^{K_{1}}\left(J_{1}, J_{1}, M_{1}, M_{1} \mid \widehat{J}_{1}\right),
\end{aligned}
$$

where

$$
\begin{aligned}
& \mathcal{A}^{r}\left(K_{0}, K_{r}, K_{1}, k, k^{\prime}\right) \\
& =\left(\alpha_{1} J_{1}\left\|Q^{(k)}\right\| \alpha_{0} J_{0}\right)\left(\alpha_{1} J_{1}\left\|Q^{\left(k^{\prime}\right)}\right\| \alpha_{0} J_{0}\right)^{*}\left\{\begin{array}{ccc}
J_{0} & K_{0} & J_{0} \\
k & K_{r} & k^{\prime} \\
J_{1} & K_{1} & J_{1}
\end{array}\right\}\left[\left(2 J_{0}+1\right)\left(2 J_{1}+1\right)(2 k+1)\left(2 K_{1}+1\right)\right]^{1 / 2}, \\
& T_{N}^{* K}\left(J, J^{\prime}, M \mid \widehat{J}\right)=(-1)^{J^{\prime}-M}\left[\frac{4 \pi}{2 J+1}\right]^{1 / 2}\left[\begin{array}{ccc}
J & J^{\prime} & K \\
M-M & 0
\end{array}\right] Y_{K N}^{*}(\theta, \phi) \\
& T_{N_{1} N_{1}^{\prime}}^{K_{1}}\left(J_{1}, J_{1}^{\prime}, M_{1}, M_{1}^{\prime} \mid \widehat{J}_{1}\right)=(-1)^{J_{1}^{\prime}-M_{1}^{\prime}}\left[\frac{2 K_{1}+1}{2 J_{1}+1}\right]^{1 / 2}\left[\begin{array}{ccc}
J_{1} & J_{1}^{\prime} & K_{1} \\
M_{1} & M_{1}^{\prime} & N_{1}^{\prime}
\end{array}\right] D_{N_{1} N_{1}^{\prime}}^{* K_{1}}\left(\widehat{J}_{1}\right) .
\end{aligned}
$$

In Eq. (12), the relation

$$
\left(\alpha_{1} J_{1}\left\|Q^{(k)}\right\| \alpha_{0} J_{0}\right)=\left[2 J_{1}+1\right]^{1 / 2}\left\langle\alpha_{1} J_{1}\left\|Q^{(k)}\right\| \alpha_{0} J_{0}\right\rangle
$$

is taken into account. In Eq. (11), $C=2 \pi, \widehat{J}$ denotes the angles of $\mathbf{J}$ with respect to the $z$ axis of laboratory frame. In the case when hyperfine structure is important, the reduced matrix element $\left(\alpha_{1} J_{1}\left\|Q^{(k)}\right\| \alpha_{0} J_{0}\right)$ should be changed by $\left(\alpha_{1} J_{1}(I) F_{1}\left\|Q^{(k)}\right\| \alpha_{0} J_{0}(I) F_{0}\right)$ in Eq. (12). A simple relation between these two matrix elements holds:

$$
\left(\alpha_{1} J_{1}(I) F_{1}\left\|Q^{(k)}\right\| \alpha_{0} J_{0}(I) F_{0}\right)=(-1)^{F_{0}-J_{1}+I+k}\left[\left(2 F_{0}+1\right)\left(2 J_{1}+1\right)\right]^{1 / 2}\left\{\begin{array}{lll}
F_{0} & k & F_{1} \\
J_{1} & I & J_{0}
\end{array}\right\}\left(\alpha_{1} J_{1}\left\|Q^{(k)}\right\| \alpha_{0} J_{0}\right) .
$$

$I$ is the spin of the nucleus. The values of $J_{0}, J_{1}$ in Eqs. (11)-(15) should be changed by $F_{0}, F_{1}$. The probability $W\left(\alpha_{0} J_{0} M_{0} \widehat{\epsilon}_{q} \mathbf{k}_{0} \rightarrow \alpha_{1} J_{1} M_{1}\right)$ equals to the cross-section (2) divided by the density of the flow of the radiation. Thus, the same expression of the cross-section can be used for the probability by changing only the definition of the constant $C$. 


\subsection{Photoionization of atoms}

The expression for the differential cross-section of the photoionization process

$$
A\left(\alpha_{0} J_{0} M_{0}\right)+h \nu\left(\epsilon_{q 1} \mathbf{k}_{0}\right) \rightarrow A^{+}\left(\alpha_{1} J_{1} M_{1}\right)+\mathrm{e}^{-}\left(\mathbf{p}_{1}, s m_{s}\right)
$$

was derived by Kupliauskiene et al. [26] in the case of dipole approximation and the levels $L S(J) I F$ of an atom. Here the ejected photoelectron has momentum $\mathbf{p}_{1}$, and the projection of its spin $s$ is indicated by $m_{s}$.

In the case when the hyperfine structure is small and taking into account of all multipoles, the general expression for the differential cross-section of Eq. (17) can be written as follows:

$$
\begin{aligned}
& \frac{\mathrm{d} \sigma\left(J_{0} M_{0} \widehat{\epsilon}_{q 1} \mathbf{k}_{01} \rightarrow J_{1} M_{1} \mathbf{p}_{1} m_{s}\right)}{\mathrm{d} \Omega_{p_{1}}} \\
& =C \sum_{K_{0}, K_{r}, K_{1}, K_{\lambda}, K_{s}, K_{j}, K, k_{1}, k_{1}^{\prime}} \mathcal{B}^{\mathrm{ph}}\left(K_{1}, K_{0}, K_{r}, K_{\lambda}, K_{s}, K_{j}, K, k_{1}, k_{1}^{\prime}\right) \\
& \times \sum_{N_{0}, N_{r}, N_{1}, N_{\lambda}, N_{j}, N, N_{s}}\left[\begin{array}{lll}
K_{1} & K_{j} & K \\
N_{1} & N_{j} & N
\end{array}\right]\left[\begin{array}{lll}
K_{\lambda} & K_{s} & K_{j} \\
N_{\lambda} & N_{s} & N_{j}
\end{array}\right]\left[\begin{array}{lll}
K_{0} & K_{r} & K \\
N_{0} & N_{r} & N
\end{array}\right] T_{N_{1} N_{1}^{\prime}}^{K_{1}}\left(J_{1}, J_{1}, M_{1}, M_{1} \mid \widehat{J}_{1}\right) \\
& \times T_{N_{0}}^{* K_{0}}\left(J_{0}, J_{0}, M_{0} \mid \widehat{J}_{0}\right) T_{N_{r}}^{* K_{r}}\left(k_{1}, k_{1}^{\prime}, q_{1} \mid \widehat{\mathbf{k}}_{01}\right) T_{N_{s}}^{K_{s}}\left(s, s, m_{s} \mid \widehat{s}\right) \sqrt{4 \pi} Y_{K_{\lambda} N_{\lambda}}\left(\widehat{\mathbf{p}}_{1}\right), \\
& \mathcal{B}^{\mathrm{ph}}\left(K_{1}, K_{0}, K_{r}, K_{\lambda}, K_{s}, K_{j}, K, k_{1}, k_{1}^{\prime}\right) \\
& =C^{\mathrm{ph}}\left(k_{1}, k_{1}^{\prime}\right) \sum_{\lambda, j, J, \lambda^{\prime}, j^{\prime}, J^{\prime}}(2 J+1)\left(2 J^{\prime}+1\right)(-1)^{\lambda^{\prime}}\left\langle\alpha_{1} J_{1} \varepsilon_{1} \lambda(j) J\left\|Q^{\left(k_{1}\right)}\right\| \alpha_{0} J_{0}\right\rangle\left\langle\alpha_{1} J_{1} \varepsilon_{1} \lambda^{\prime}\left(j^{\prime}\right) J^{\prime}\left\|Q^{\left(k_{1}^{\prime}\right)}\right\| \alpha_{0} J_{0}\right\rangle^{*} \\
& \times\left[\left(2 J_{0}+1\right)\left(2 K_{j}+1\right)\left(\left(2 J_{1}+1\right)\left(2 k_{1}+1\right)(2 s+1)(2 \lambda+1)\left(2 \lambda^{\prime}+1\right)(2 j+1)\left(2 j^{\prime}+1\right)\right]^{1 / 2}\right. \\
& \times\left[\begin{array}{ccc}
\lambda & \lambda^{\prime} & K_{\lambda} \\
0 & 0 & 0
\end{array}\right]\left\{\begin{array}{ccc}
J_{0} & K_{0} & J_{0} \\
k_{1} & K_{r} & k_{1}^{\prime} \\
J^{\prime} & K & J
\end{array}\right\}\left\{\begin{array}{ccc}
J_{1} & K_{1} & J_{1} \\
j^{\prime} & K_{j} & j \\
J^{\prime} & K & J
\end{array}\right\}\left\{\begin{array}{ccc}
\lambda^{\prime} & K_{\lambda} \lambda \\
s & K_{s} & s \\
j^{\prime} & K_{j} & j
\end{array}\right\} .
\end{aligned}
$$

In the case of the photoionization as the first step process, the expression for the cross-section should be modified (see Section 2.3). It acquires the form

$$
\begin{aligned}
& \frac{\mathrm{d} \sigma_{K_{1} N_{1}}\left(\alpha_{0} J_{0} M_{0} \widehat{\epsilon}_{q 1} \mathbf{k}_{01} \rightarrow \alpha_{1} J_{1} \mathbf{p}_{1} m_{s}\right)}{\mathrm{d} \Omega_{p_{1}}} \\
& =C \sum_{K_{0}, K_{r}, K_{\lambda}, K_{s}, K_{j}, K, k_{1}, k_{1}^{\prime}} \mathcal{B}^{\mathrm{ph}}\left(K_{1}, K_{0}, K_{r}, K_{\lambda}, K_{s}, K_{j}, K, k_{1}, k_{1}^{\prime}\right)\left[\frac{2 K_{1}+1}{2 J_{1}+1}\right]^{1 / 2} \\
& \quad \times \sum_{N_{0}, N_{r}, N_{\lambda}, N_{j}, N, N_{s}}\left[\begin{array}{c}
K_{1} K_{j} K \\
N_{1} N_{j} N
\end{array}\right]\left[\begin{array}{l}
K_{\lambda} K_{s} K_{j} \\
N_{\lambda} N_{s} N_{j}
\end{array}\right]\left[\begin{array}{c}
K_{0} K_{r} K \\
N_{0} N_{r} N
\end{array}\right] \\
& \quad \times T_{N_{0}}^{* K_{0}}\left(J_{0}, J_{0}, M_{0} \mid \widehat{J}_{0}\right) T_{N_{r}}^{* K_{r}}\left(k_{1}, k_{1}^{\prime}, q_{1} \mid \widehat{\mathbf{k}}_{01}\right) T_{N_{s}}^{K_{s}}\left(s, s, m_{s} \mid \widehat{s}\right) \sqrt{4 \pi} Y_{K_{\lambda} N_{\lambda}}\left(\widehat{\mathbf{p}}_{1}\right) .
\end{aligned}
$$

Further the general expressions (18)-(20) can be used to obtain some special expressions for specific experimental conditions also investigated by other authors with the help of the density matrix formalism, e. g., the expressions for the angular distribution and spin polarization of photoelectrons in the case of nonpolarized atom. 


\subsection{One- and multistep processes}

The excitation or ionization of an atom by laser or other electromagnetic radiation is often used to prepare it in a polarized state for further investigation. Then the magnetic state of an atom or ion in the final state $J_{1} M_{1}$ is not observed, and summation of $M_{1}$ has to be performed coherently. In this case, the excitation (1) is the first step of the multistep process while the second step is

$$
A^{*}\left(\alpha_{1} J_{1} M_{1}\right)+b(\alpha) \rightarrow A\left(\alpha_{2} J_{2} M_{2}\right)+b^{\prime}\left(\alpha^{\prime}\right) .
$$

Here $b(\alpha)$ stands for impacting particle or electromagnetic radiation in the state $\alpha$ and $b^{\prime}\left(\alpha^{\prime}\right)$ indicates ionized and emitted one or more particles. In two-step approximation, the probability of both processes (1) and (21) can be written as coherent sum since the projection $M_{1}$ cannot be observed [18]:

$$
\begin{aligned}
W\left(\alpha_{0} J_{0} M_{0} \hat{\epsilon} \mathbf{k}_{0} \rightarrow \alpha_{1} J_{1} \alpha \rightarrow \alpha_{2} J_{2} M_{2} \alpha^{\prime}\right)= & C^{\prime}\left|\sum_{M_{1}}\left\langle\alpha_{2} J_{2} M_{2} \alpha^{\prime}\left|H_{2}\right| \alpha_{1} J_{1} M_{1} \alpha\right\rangle\left\langle\alpha_{1} J_{1} M_{1}\left|H_{1}\right| \alpha_{0} J_{0} M_{0}\right\rangle\right|^{2} \\
= & C^{\prime} \sum_{M_{1}, M_{1}^{\prime}}\left\langle\alpha_{2} J_{2} M_{2} \alpha^{\prime}\left|H_{2}\right| \alpha_{1} J_{1} M_{1} \alpha\right\rangle\left\langle\alpha_{2} J_{2} M_{2} \alpha^{\prime}\left|H_{2}\right| \alpha_{1} J_{1} M_{1}^{\prime} \alpha\right\rangle^{*} \\
& \times\left\langle\alpha_{1} J_{1} M_{1}\left|H_{1}\right| \alpha_{0} J_{0} M_{0}\right\rangle\left\langle\alpha_{1} J_{1} M_{1}^{\prime}\left|H_{1}\right| \alpha_{0} J_{0} M_{0}\right\rangle^{*}
\end{aligned}
$$

The matrix elements in Eq. (22) are defined similar to Eq. (10) allowing the measurement in the direction different from that used for evaluation. $H_{1}$ and $H_{2}$ are the operators of the interaction in the first and second processes, respectively. Then the product of two Wigner rotation matrices coming up from the matrix element and its complex conjugate in Eq. (22) should be replaced by

$$
D_{\widetilde{M}_{1} M_{1}}^{J_{1}}\left(\widehat{J}_{1}\right) D_{\widetilde{M}_{1}^{\prime} M_{1}^{\prime}}^{* J_{1}^{\prime}}\left(\widehat{J}_{1}\right)=\sum_{K_{1}, N_{1}, N_{1}^{\prime}}\left[\begin{array}{ccc}
J_{1}^{\prime} & K_{1} & J_{1} \\
\widetilde{M}_{1}^{\prime} & N_{1} & \widetilde{M}_{1}
\end{array}\right] T_{N_{1} N_{1}^{\prime}}^{* K_{1}}\left(J_{1}, J_{1}^{\prime}, M_{1}, M_{1}^{\prime} \mid \widehat{J}_{1}\right),
$$

and the summation over $M_{1}$ and $M_{1}^{\prime}$ in Eq. (22) is possible to be carried out. From the examination of the expressions (29) in [44] and (11) and (18) of the present work for both terms in Eq. (22) it follows that only the tensors $T_{N_{1} N_{1}^{\prime}}^{K_{1}}\left(J_{1}, J_{1}^{\prime}, M_{1}, M_{1}^{\prime} \mid \widehat{J}_{1}\right)$ depend on $M_{1}$ and $M_{1}^{\prime}$. The sum over $M_{1}, M_{1}^{\prime}$ of the product of these tensors is equal to

$$
\begin{aligned}
& \sum_{M_{1}, M_{1}^{\prime}} T_{N_{1} N}^{K_{1}}\left(J_{1}, J_{1}, M_{1}, M_{1}^{\prime} \mid \widehat{J}_{1}\right) T_{N_{1}^{\prime} N^{\prime}}^{* K_{1}^{\prime}}\left(J_{1}, J_{1}, M_{1}, M_{1}^{\prime} \mid \widehat{J}_{1}\right) \\
& =\frac{\sqrt{\left(2 K_{1}+1\right)\left(2 K_{1}^{\prime}+1\right)}}{2 J_{1}+1} D_{N_{1} N}^{* K_{1}}(\widehat{J}) D_{N_{1}^{\prime} N^{\prime}}^{K^{\prime}}(\widehat{J}) \sum_{M_{1}, M_{1}^{\prime}}(-1)^{2 J_{1}-2 M_{1}^{\prime}}\left[\begin{array}{ccc}
J_{1} & J_{1} & K_{1} \\
M_{1} & M_{1}^{\prime} & N
\end{array}\right]\left[\begin{array}{ccc}
J_{1} & J_{1} & K_{1}^{\prime} \\
M_{1} & M_{1}^{\prime} & N^{\prime}
\end{array}\right] \\
& =\frac{2 K_{1}+1}{2 J_{1}+1} D_{N_{1} N}^{* K_{1}}(\widehat{J}) D_{N_{1}^{\prime} N}^{K_{1}}(\widehat{J}) \delta\left(K_{1}, K_{1}^{\prime}\right) \delta\left(N, N^{\prime}\right) .
\end{aligned}
$$

Then the quantization axis can be chosen along the $z$ axis of the laboratory coordinate system, and $D_{N_{1} N}^{* K_{1}}(0,0,0) D_{N_{1}^{\prime} N}^{K_{1}}(0,0,0)=\delta\left(N_{1}, N\right) \delta\left(N_{1}^{\prime}, N\right)$. The square root of the multiplier $\left(2 K_{1}+1\right) /\left(2 J_{1}+1\right)$ is convenient to attribute to both terms of the following expression:

$$
\begin{aligned}
& W\left(\alpha_{0} J_{0} M_{0} \hat{\epsilon} \mathbf{k}_{0} \rightarrow \alpha_{1} J_{1} \alpha \rightarrow \alpha_{2} J_{2} M_{2} \alpha^{\prime}\right) \\
& \quad=\sum_{K_{1}, N_{1}} W_{K_{1} N_{1}}\left(\alpha_{0} J_{0} M_{0} \widehat{\epsilon} \mathbf{k}_{0} \rightarrow \alpha_{1} J_{1} \alpha\right) W_{K_{1} N_{1}}^{A}\left(\alpha_{1} J_{1} \alpha \rightarrow \alpha_{2} J_{2} M_{2} \alpha^{\prime}\right) .
\end{aligned}
$$


Here the sum over $M_{1}, M_{1}^{\prime}$ in Eq. (22) is replaced by the sum over $K_{1}, N_{1}$, i. e. the probability of the two-step process is expanded as the sum of state multipoles. For example, the expressions for the photoexcitation probability (11) and that of the second process slightly change. For the excitation probability, it is

$$
\begin{aligned}
W_{K_{1} N_{1}}\left(\alpha_{0} J_{0} M_{0} \widehat{\epsilon}_{q} \mathbf{k}_{0} \rightarrow \alpha_{1} J_{1}\right)= & \frac{C}{\left[2 J_{1}+1\right]^{1 / 2}} \sum_{K_{0}, K_{r}, k, k^{\prime}} B^{r}\left(K_{0}, K_{r}, K_{1}, k, k^{\prime}\right) \sum_{N_{0}, N_{r}, q}\left[\begin{array}{l}
K_{0} K_{r} K_{1} \\
N_{0} N_{r} N_{1}
\end{array}\right] \\
& \times T_{N_{0}}^{* K_{0}}\left(J_{0}, J_{0}, M_{0} \mid \widehat{J}_{0}\right) T_{N_{r}}^{* K_{r}}\left(k, k^{\prime}, q \mid \widehat{k}_{0}\right), \\
W_{00}\left(\alpha_{0} J_{0} k \rightarrow \alpha_{1} J_{1}\right)= & \frac{C}{\left(2 J_{0}+1\right)(2 k+1)\left[2 J_{1}+1\right]^{1 / 2}}\left|\left\langle\alpha_{1} J_{1}|| Q^{(k)} \| \alpha_{0} J_{0}\right\rangle\right|^{2} .
\end{aligned}
$$

The expression for the second term in Eq. (25) depends on the second-step process. In the case of the Auger decay, it is presented by Kupliauskienè and Tutlys (see Eq. (4) in [45]).

The proposed method is easy to generalize for multistep process when intermediate states are not observed. In the case of a three-step process where the fluorescence radiation of the doubly charged ion formed following the photoionization of an atom and Auger decay of the singly charged ion is registered, the summation over intermediate states gives

$$
\begin{aligned}
& \sum_{M_{1}, M_{1}^{\prime}, M_{2}, M_{2}^{\prime}} W^{1}\left(J_{0} M_{0} \mathbf{k}_{1} \rightarrow J_{1} M_{1} M_{1}^{\prime} \mathbf{p}_{1} m_{1}\right) W^{2}\left(J_{1} M_{1} M_{1}^{\prime} \mathbf{p}_{1} m_{1} \rightarrow J_{2} M_{2} M_{2}^{\prime} \mathbf{p}_{2} m_{2}\right) \\
& \quad \times W^{3}\left(J_{2} M_{2} M_{2}^{\prime} \mathbf{p}_{2} m_{2} \rightarrow J_{3} M_{3} \mathbf{k}_{2}\right) \\
& =\sum_{K_{1}, N_{1}, K_{2}, N_{2}} W_{K_{1} N_{1}}^{1}\left(J_{0} M_{0} \mathbf{k}_{1} \rightarrow J_{1} \mathbf{p}_{1} m_{1}\right) \frac{2 K_{1}+1}{2 J_{1}+1} W_{K_{1} N_{1} K_{2} N_{2}}^{2}\left(J_{1} \mathbf{p}_{1} m_{1} \rightarrow J_{2} \mathbf{p}_{2} m_{2}\right) \\
& \quad \times \frac{2 K_{2}+1}{2 J_{2}+1} W_{K_{2} N_{2}}^{3}\left(J_{2} \mathbf{p}_{2} m_{2} \rightarrow J_{3} M_{3} \mathbf{k}_{2}\right) .
\end{aligned}
$$

The square root of each multiplier $(2 K+1) /(2 J+1)$ in Eq. (28) is also convenient to attribute to both neighbouring terms.

\section{Electron-atom interactions}

\subsection{Excitation of atoms by electrons}

The process of the excitation of polarized atoms by polarized electrons can be written as follows:

$$
A\left(\alpha_{0} J_{0} M_{0}\right)+\mathrm{e}^{-}\left(\mathbf{p}_{0} m_{0}\right) \rightarrow A\left(\alpha_{1} J_{1} M_{1}\right)+\mathrm{e}^{-}\left(\mathbf{p}_{1} m_{1}\right) .
$$

The expression for the differential cross-section of the process (29) is easy to obtain by the method described in $[26,44]$ and is as follows:

$$
\begin{aligned}
& \frac{\mathrm{d}^{2} \sigma\left(\alpha_{0} J_{0} M_{0} \mathbf{p}_{0} m_{0} \rightarrow \alpha_{1} J_{1} M_{1} \mathbf{p}_{1} m_{1}\right)}{\mathrm{d} \varepsilon_{2} \mathrm{~d} \Omega_{1}} \\
& =4 \pi C \sum_{K, K_{0}, K_{0}^{\prime}, K_{\lambda 0}, K_{s 0}, K_{1},} \mathcal{B}^{\mathrm{ex}}\left(K_{0}, K_{0}^{\prime}, K_{1}, K_{1}^{\prime}, K_{\lambda 0}, K_{s 0}, K_{\lambda 1}, K_{s 1}, K\right) \\
& K_{1}^{\prime}, K_{\lambda 1}, K_{s 1}
\end{aligned}
$$




$$
\begin{aligned}
& \times \sum_{N_{0}, N_{0}^{\prime}, N_{\lambda 0}, N_{s 0}, N_{1},}\left[\begin{array}{lll}
K_{\lambda 0} & K_{s 0} & K_{0}^{\prime} \\
N_{\lambda 0} & N_{s 0} & N_{0}^{\prime}
\end{array}\right]\left[\begin{array}{lll}
K_{0} & K_{0}^{\prime} & K \\
N_{0} & N_{0}^{\prime} & N
\end{array}\right]\left[\begin{array}{lll}
K_{1} & K^{\prime} & K \\
N_{1} & N^{\prime} & N
\end{array}\right]\left[\begin{array}{lll}
K_{1} & K_{1}^{\prime} & K \\
N_{1} & N_{1}^{\prime} & N
\end{array}\right]\left[\begin{array}{lll}
K_{\lambda 1} & K_{s 1} & K_{1}^{\prime} \\
N_{\lambda 1} & N_{s 1} & N_{1}^{\prime}
\end{array}\right] \\
& N_{1}^{\prime}, N_{\lambda 1}, N_{s 1}, N \\
& \times Y_{K_{\lambda 0} N_{\lambda 0}}^{*}\left(\widehat{p}_{0}\right) Y_{K_{\lambda 1} N_{\lambda 1}}\left(\widehat{p}_{1}\right) T_{N_{0}}^{* K_{0}}\left(J_{0}, J_{0}, M_{0} \mid \widehat{J}_{0}\right) T_{N_{1}}^{K_{1}}\left(J_{1}, J_{1}, M_{1} \mid \widehat{J}_{1}\right) T_{N_{s 0}}^{* K_{s 0}}\left(s, s, m_{0} \mid \widehat{s}\right) T_{N_{s 1}}^{K_{s 1}}\left(s, s, m_{1} \mid \widehat{s}\right)
\end{aligned}
$$$$
\mathcal{B}^{\mathrm{ex}}\left(K_{0}, K_{0}^{\prime}, K_{1}, K_{1}^{\prime}, K_{\lambda 0}, K_{s 0}, K_{\lambda 1}, K_{s 1}, K\right)
$$$$
=\sum_{\lambda_{0}, \lambda_{0}^{\prime}, \lambda_{1}, \lambda_{1}^{\prime}, j_{0}, j_{0}^{\prime}, j_{1}, j_{1}^{\prime}, J, J^{\prime}}(2 J+1)\left(2 J^{\prime}+1\right)(2 K+1)(2 s+1)(-1)^{\lambda_{0}^{\prime}+\lambda_{1}^{\prime}}
$$$$
\times\left\langle\alpha_{1} J_{1}, \varepsilon_{1} \lambda_{1}\left(j_{1}\right) J\|H\| \alpha_{0} J_{0}, \varepsilon_{0} \lambda_{0}\left(j_{0}\right) J\right\rangle\left\langle\alpha_{1} J_{1}, \varepsilon_{1} \lambda_{1}^{\prime}\left(j_{1}^{\prime}\right) J^{\prime}\|H\| \alpha_{0} J_{0}, \varepsilon_{0} \lambda_{0}^{\prime}\left(j_{0}^{\prime}\right) J^{\prime}\right\rangle^{*}
$$$$
\times\left[\left(2 \lambda_{0}+1\right)\left(2 \lambda_{0}^{\prime}+1\right)\left(2 \lambda_{1}+1\right)\left(2 \lambda_{1}^{\prime}+1\right)\left(2 j_{0}+1\right)\left(2 j_{0}^{\prime}+1\right)\right.
$$$$
\left.\times\left(2 j_{1}+1\right)\left(2 j_{1}^{\prime}+1\right)\left(2 J_{0}+1\right)\left(2 J_{1}+1\right)\left(2 K_{0}^{\prime}+1\right)\left(2 K_{1}^{\prime}+1\right)\right]^{1 / 2}
$$$$
\times\left[\begin{array}{ccc}
\lambda_{0} & \lambda_{0}^{\prime} & K_{\lambda 0} \\
0 & 0 & 0
\end{array}\right]\left[\begin{array}{ccc}
\lambda_{1} & \lambda_{1}^{\prime} & K_{\lambda 1} \\
0 & 0 & 0
\end{array}\right]\left\{\begin{array}{ccc}
J_{0} & K_{0} & J_{0} \\
j_{0}^{\prime} & K_{0}^{\prime} & j_{0} \\
J^{\prime} & K & J
\end{array}\right\}\left\{\begin{array}{ccc}
\lambda_{0}^{\prime} & K_{\lambda 0} & \lambda_{0} \\
s & K_{s 0} & s \\
j_{0}^{\prime} & K_{0}^{\prime} & j_{0}
\end{array}\right\}\left\{\begin{array}{cccc}
\lambda_{1}^{\prime} & K_{\lambda 1} & \lambda_{1} \\
s & K_{s 1} & s \\
j_{1}^{\prime} & K_{1}^{\prime} & j_{1}
\end{array}\right\}\left\{\begin{array}{ccc}
J_{1} & K_{1} & J_{1} \\
j_{1}^{\prime} & K_{1}^{\prime} & j_{1} \\
J & K & J^{\prime}
\end{array}\right\} .
$$

In Eq. (31), $\left\langle\alpha_{1} J_{1}, \varepsilon_{1} \lambda_{1}\left(j_{1}\right) J\|H\| \alpha_{0} J_{0}, \varepsilon_{0} \lambda_{0}\left(j_{0}\right) J\right\rangle$ is the reduced matrix element of the electrostatic interaction. If the excitation of atoms by electrons is used as the first step process for the preparation of atoms in polarized excited states for the next step process, the projections $M_{1}$ are not registered. Then the summation over $M_{1}$ should be performed following the recommendations of Section 2.3.

\subsection{Ionization of atoms by electrons}

If neither the projection quantum numbers of the incident electron nor the target are resolved, the differential cross-section for the ionization of atoms by electrons is simply a scalar with respect to the joint rotation of the incoming and outgoing electron momenta $\mathbf{p}_{0}, \mathbf{p}_{1}$, and $\mathbf{p}_{2}$, respectively. This basic symmetry is destroyed by an initial orientation and/or alignment of the target, and can supply more information about the elementary processes in such diverse fields as discharge and plasma physics [47], fusion physics [8], and the physics and chemistry of the upper atmosphere [48]. For the interpretation of recently measured ionization cross-sections of polarized Na atoms $[48,49]$, the expressions enabling one to describe the polarization states of all particles taking part in the process are necessary.

Recently the general expression for the description of the ionization of polarized atoms by polarized electrons

$$
A\left(\alpha_{0} J_{0} M_{0}\right)+\mathrm{e}^{-}\left(\mathbf{p}_{0} m_{0}\right) \rightarrow A^{+}\left(\alpha_{1} J_{1} M_{1}\right)+\mathrm{e}^{-}\left(\mathbf{p}_{2} m_{2}\right)+\mathrm{e}^{-}\left(\mathbf{p}_{1} m_{1}\right)
$$

was obtained by Kupliauskienè and Glemža [46]. In Eq. (32), $\mathbf{p}_{i}$ denotes the momentum of the electron in the initial $(i=0)$ and final $(i=1,2)$ states, $m_{i}$ indicates the projection of an electron spin. The fine structure splitting was assumed larger than hyperfine one. The expression for the cross-section was derived by using the graphical technique of the angular momentum [1] and is as follows:

$$
\begin{aligned}
& \frac{\mathrm{d}^{3} \sigma\left(\alpha_{0} J_{0} M_{0} \mathbf{p}_{0} m_{0} \rightarrow \alpha_{1} J_{1} M_{1} \mathbf{p}_{2} m_{2} \mathbf{p}_{1} m_{1}\right)}{\mathrm{d} \varepsilon_{2} \mathrm{~d} \Omega_{1} \mathrm{~d} \Omega_{2}}
\end{aligned}
$$

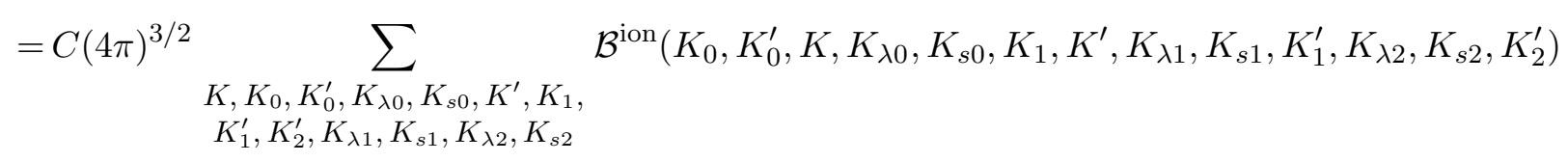




$$
\begin{aligned}
& \times \sum_{N, N_{0}, N_{0}^{\prime}, N_{\lambda 0}, N_{s 0}, N^{\prime}, N_{1},}\left[\begin{array}{lll}
K_{\lambda 0} & K_{s 0} & K_{0}^{\prime} \\
N_{\lambda 0} & N_{s 0} & N_{0}^{\prime}
\end{array}\right]\left[\begin{array}{lll}
K_{0} & K_{0}^{\prime} & K \\
N_{0} & N_{0}^{\prime} & N
\end{array}\right]\left[\begin{array}{lll}
K_{1} & K^{\prime} & K \\
N_{1} & N^{\prime} & N
\end{array}\right]\left[\begin{array}{lll}
K_{2}^{\prime} & K_{1}^{\prime} & K^{\prime} \\
N_{2}^{\prime} & N_{1}^{\prime} & N^{\prime}
\end{array}\right]\left[\begin{array}{lll}
K_{\lambda 1} & K_{s 1} & K_{1}^{\prime} \\
N_{\lambda 1} & N_{s 1} & N_{1}^{\prime}
\end{array}\right] \\
& N_{1}^{\prime}, N_{2}^{\prime}, N_{\lambda 1}, N_{s 1}, N_{\lambda 2}, N_{s 2} \\
& \times\left[\begin{array}{ccc}
K_{\lambda 2} & K_{s 2} & K_{2}^{\prime} \\
N_{\lambda 2} & N_{s 2} & N_{2}
\end{array}\right] Y_{K_{\lambda 0} N_{\lambda 0}}^{*}\left(\widehat{p}_{0}\right) Y_{K_{\lambda 1} N_{\lambda 1}}\left(\widehat{p}_{1}\right) Y_{K_{\lambda 2} N_{\lambda 2}}\left(\widehat{p}_{2}\right) T_{N_{0}}^{* K_{0}}\left(J_{0}, J_{0}, M_{0} \mid \widehat{J}_{0}\right) T_{N_{1}}^{K_{1}}\left(J_{1}, J_{1}, M_{1} \mid \widehat{J}_{1}\right) \\
& \times T_{N_{s 0}}^{* K_{s 0}}\left(s, s, m_{0} \mid \widehat{s}\right) T_{N_{s 1}}^{K_{s 1}}\left(s, s, m_{1} \mid \widehat{s}\right) T_{N_{s 2}}^{K_{s 2}}\left(s, s, m_{2} \mid \widehat{s}\right), \\
& \mathcal{B}^{\text {ion }}\left(K_{0}, K_{0}^{\prime}, K, K_{\lambda 0}, K_{s 0}, K_{1}, K^{\prime}, K_{\lambda 1}, K_{s 1}, K_{1}^{\prime}, K_{\lambda 2}, K_{s 2}, K_{2}^{\prime}\right) \\
& =\sum_{\substack{\lambda_{0}, \lambda_{0}^{\prime}, \lambda_{1}, \lambda_{1}^{\prime}, \lambda_{2}, \lambda_{2}^{\prime}, j_{0}, j_{0}^{\prime}, j_{1}, j_{1}^{\prime}, j_{2}, j_{2}^{\prime}, J, J^{\prime}, j, j^{\prime}}}(2 J+1)\left(2 J^{\prime}+1\right)(2 K+1)(2 s+1)(-1)^{\lambda_{0}^{\prime}+\lambda_{1}^{\prime}+\lambda_{2}^{\prime}} \\
& \times\left\langle\alpha_{1} J_{1}, \varepsilon_{2} \lambda_{2}\left(j_{2}\right) \varepsilon_{1} \lambda_{1}\left(j_{1}\right) j, J\|H\| \alpha_{0} J_{0}, \varepsilon_{0} \lambda_{0}\left(j_{0}\right) J\right\rangle\left\langle\alpha_{1} J_{1}, \varepsilon_{2} \lambda_{2}^{\prime}\left(j_{2}^{\prime}\right) \varepsilon_{1} \lambda_{1}^{\prime}\left(j_{1}^{\prime}\right) j^{\prime}, J^{\prime}\|H\| \alpha_{0} J_{0}, \varepsilon_{0} \lambda_{0}^{\prime}\left(j_{0}^{\prime}\right) J^{\prime}\right\rangle^{*} \\
& \times\left[(2 s+1)\left(2 \lambda_{0}+1\right)\left(2 \lambda_{0}^{\prime}+1\right)\left(2 \lambda_{1}+1\right)\left(2 \lambda_{1}^{\prime}+1\right)\left(2 \lambda_{2}+1\right)\left(2 \lambda_{2}^{\prime}+1\right)\left(2 j_{0}+1\right)\left(2 j_{0}^{\prime}+1\right)\right. \\
& \times\left(2 j_{1}+1\right)\left(2 j_{1}^{\prime}+1\right)\left(2 j_{2}+1\right)\left(2 j_{2}^{\prime}+1\right)(2 j+1)\left(2 j^{\prime}+1\right)\left(2 J_{0}+1\right)\left(2 J_{1}+1\right)\left(2 K_{0}^{\prime}+1\right)\left(2 K_{1}^{\prime}+1\right) \\
& \left.\times\left(2 K^{\prime}+1\right)\left(2 K_{2}^{\prime}+1\right)\right]^{1 / 2}\left[\begin{array}{ccc}
\lambda_{0} & \lambda_{0}^{\prime} & K_{\lambda 0} \\
0 & 0 & 0
\end{array}\right]\left[\begin{array}{ccc}
\lambda_{1} & \lambda_{1}^{\prime} & K_{\lambda 1} \\
0 & 0 & 0
\end{array}\right]\left[\begin{array}{ccc}
\lambda_{2} & \lambda_{2}^{\prime} & K_{\lambda 2} \\
0 & 0 & 0
\end{array}\right]
\end{aligned}
$$

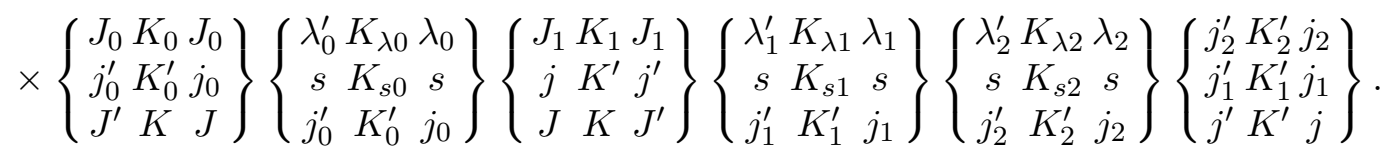

The reduced matrix element of the electrostatic interaction in Eq. (34) is defined in [2]. In the case of the inner-shell ionization of atoms, the state of the ion is not stable and decays via radiative or Auger transition. Then the expression (33) should be modified following the recommendations of Section 2.3 as it was pointed out in Section 2.2.

The expression (33) for the electron-impact ionization can also be used for the investigation of the ionization of atoms by protons and highly charged ions. Then the expressions for the cross-section and reduced matrix element should suffer some changes. The alignment parameters for $L_{3}$-subshell of $\mathrm{Cd}$ and $\mathrm{Sb}$ atoms were obtained by observing the degree of polarization of the $L_{1}$-lines excited by proton impact [50].

\subsection{Radiative recombination}

The process of the radiative recombination of polarized ions with polarized electrons can be written as follows:

$$
A^{n+}\left(\alpha_{0} J_{0} M_{0}\right)+\mathrm{e}^{-}\left(\mathbf{p}_{0} m_{0}\right) \rightarrow A^{(n-1)+}\left(\alpha_{1} J_{1} M_{1}\right)+h \nu\left(\epsilon_{q} \mathbf{k}_{0}\right) .
$$

From the relation of detailed balance, it follows that the cross-section of the radiative recombination $\sigma_{f \rightarrow i}^{\mathrm{rr}}\left(E_{0}\right)$ is related to the cross-section of the photoionization $\sigma_{i \rightarrow f}^{\mathrm{ph}}\left(E_{1}\right)$ thought detailed balance (Milne relation) as

$$
\sigma_{f \rightarrow i}^{\mathrm{rr}}\left(E_{0}\right)=\frac{\left(\alpha E_{1}\right)^{2}}{2 E_{0}} \frac{g_{i}}{g_{f}} \sigma_{i \rightarrow f}^{\mathrm{ph}}\left(E_{1}\right) .
$$

Here $E_{0}$ and $E_{1}$ are the energy of an electron and emitted photon, respectively, $g_{i}$ and $g_{f}$ are the statistical weights of the initial and final states, $\alpha$ is the fine structure constant, and $E_{1}=E_{0}+I_{p}$ ( $I_{p}$ is the ionization energy). Then 
the general expression for the differential radiative recombination cross-section can be written in the following form by using Eq. (18):

$$
\begin{aligned}
& \frac{\mathrm{d} \sigma\left(J_{0} M_{0} \mathbf{p}_{1} m_{s} \rightarrow J_{1} M_{1} \widehat{\epsilon}_{q 1} \mathbf{k}_{01}\right)}{\mathrm{d} \Omega} \\
& =C \sqrt{4 \pi} \sum_{K_{0}, K_{r}, K_{1}, K_{s}, K_{j}, K, k_{1}, k_{1}^{\prime}} B^{\mathrm{ph}}\left(K_{0}, K_{1}, K_{r}, K_{\lambda}, K_{s}, K_{j}, K, k_{1}, k_{1}^{\prime}\right) \\
& \quad \times \sum_{N_{0}, N_{r}, N_{1}, N_{s}, N_{j}, N}\left[\begin{array}{l}
K_{0} K_{j} K \\
N_{0} N_{j} N
\end{array}\right]\left[\begin{array}{l}
K_{\lambda} K_{s} K_{j} \\
N_{\lambda} N_{s} N_{j}
\end{array}\right]\left[\begin{array}{l}
K_{1} K_{r} K \\
N_{1} N_{r} N
\end{array}\right] Y_{K_{\lambda}, N_{\lambda}}^{*}\left(\widehat{\mathbf{p}}_{1}\right) T_{N_{0}}^{* K_{0}}\left(J_{0}, J_{0}, M_{0} \mid \widehat{J}_{0}\right) \\
& \quad \times T_{N_{r}}^{K_{r}}\left(k_{1}, k_{1}^{\prime}, q_{1} \mid \widehat{\mathbf{k}}_{01}\right) T_{N_{s}}^{* K_{s}}\left(s, s, m_{s} \mid \widehat{s}\right) T_{N_{1} N_{1}^{\prime}}^{K_{1}}\left(J_{1}, J_{1}, M_{1}, M_{1}^{\prime} \mid \widehat{J}_{1}\right) .
\end{aligned}
$$

Here $C=\left(\alpha^{2} E_{1}^{2}\right) / E_{0}$.

If a recombined ion arises in the excited state, it can suffer the radiative decay to a lower excited or ground state by emitting a photon. The polarization state of this photon depends on the states of the ion and electron. For the description of the polarization characteristics of the second photon, the expression for the radiative recombination cross-section as that of the first-step process is necessary. It can be obtained following the recommendations of Section 2.3.

\section{Decay of excited atoms}

In the case of the registration of the decay products of the formed ion, much information can be gained not only about the structure of the system under investigation but also about the process itself and many-body interactions. Here the Auger decay process is often used [51]. But in the case of the creation of a vacancy in the outermost closed shell of atoms containing one valence electron, the radiative transition is the only way of its decay. The fluorescence and Auger decay of an excited ion or atom are the processes of the second step following inner-shell excitation or ionization of the atom. In the present section, the expressions for the radiative and Auger decay as the second step process will be presented. These expressions were obtained by applying atomic theory methods [2,27] and the graphical technique of the angular momentum [1].

\subsection{Radiative decay}

The radiative decay of an atom or ion is the inverse process to the photoexcitation and can be written in the form

$$
A\left(\alpha_{1} J_{1} M_{1}\right) \rightarrow A\left(\alpha_{2} J_{2} M_{2}\right)+h \nu\left(\widehat{\epsilon}_{q 2} \mathbf{k}_{02}\right) .
$$

The general expression for the probability of the fluorescence following the photoionization of polarized atoms was obtained by Kupliauskiene and Tutlys [52]. In the case of fluorescence as the second step process, the initial state $M_{1}$ is not registered. Then, the expression for the radiative transition probability is as follows:

$$
\begin{aligned}
& \frac{\mathrm{d} W_{K_{1} N_{1}}^{r}\left(\alpha_{1} J_{1} \rightarrow \alpha_{2} J_{2} M_{2} \widehat{\epsilon}_{q 2} \mathbf{k}_{02}\right)}{\mathrm{d} \Omega_{2}} \\
& \quad=\sum_{K_{r}^{\prime}, K_{2}, k_{2}, k_{2}^{\prime}} \mathcal{A}\left(K_{1}, K_{r}^{\prime}, K_{2}, k_{2}, k_{2}^{\prime}\right) \sum_{N_{r}^{\prime}, N_{2}}\left[\begin{array}{c}
K_{1} K_{r}^{\prime} K_{2} \\
N_{1} N_{r}^{\prime} N_{2}
\end{array}\right] T_{N_{2}}^{K_{2}}\left(J_{2}, J_{2}, M_{2} \mid \widehat{J}_{2}\right) T_{N_{r}^{\prime}}^{K_{r}^{\prime}}\left(k_{2}, k_{2}^{\prime}, q_{2} \mid \widehat{\mathbf{k}}_{02}\right), \\
& \mathcal{A}\left(K_{1}, K_{r}^{\prime}, K_{2}, k_{2}, k_{2}^{\prime}\right) \\
& \quad=C\left(k_{2}, k_{2}^{\prime}\right)\left(\alpha_{2} J_{2}\left\|Q^{\left(k_{2}\right)}\right\| \alpha_{1} J_{1}\right)\left(\alpha_{2} J_{2}\left\|Q^{\left(k_{2}^{\prime}\right)}\right\| \alpha_{1} J_{1}\right)^{*}\left[\frac{\left(2 K_{1}+1\right)\left(2 J_{2}+1\right)\left(2 k_{2}+1\right)}{2 K_{2}+1}\right]^{1 / 2}\left\{\begin{array}{l}
J_{1} K_{1} J_{1} \\
k_{2} K_{r}^{\prime} k_{2}^{\prime} \\
J_{2} K_{2} J_{2}
\end{array}\right\} .
\end{aligned}
$$


The reduced matrix element in Eq. (40) is equal to

$$
\left(\alpha_{2} J_{2}\left\|Q^{\left(k_{2}\right)}\right\| \alpha_{1} J_{1}\right)=\left(2 J_{2}+1\right)^{1 / 2} k_{02}\left\langle\alpha_{2} J_{2}\left\|Q^{\left(k_{2}\right)}\right\| \alpha_{1} J_{1}\right\rangle
$$

and is defined by Eq. (4). The constant $C\left(k_{2}, k_{2}^{\prime}\right)$ depends on the multipolarity of the transition operator. In the case of electrical dipole transition, $C(1,1)=\alpha^{3} \Delta E^{3} /(2 \pi)$, where $\alpha$ is the fine structure constant and $\Delta E$ is the difference of the energies (in a.u.) of the initial and final states.

\subsection{Auger decay}

Usually Auger decay is the second step process

$$
A^{+}\left(\alpha_{1} J_{1} M_{1}\right) \rightarrow A^{2+}\left(\alpha_{2} J_{2} M_{2}\right)+\mathrm{e}^{-}\left(\mathbf{p}_{2}, m_{s}^{\prime}\right)
$$

following inner-shell excitation or ionization of atoms and ions. The general expression for the probability of Eq. (42) was obtained by Kupliauskienè and Tutlys $[44,45]$ in the case when the Auger transition follows the photoionization of an atom.

When the state of the intermediate ion is not registered, the Auger transition probability acquires the form [45]

$$
\begin{aligned}
& \frac{\mathrm{d} W_{K_{1} N_{1}}\left(\alpha_{1} J_{1} \rightarrow \alpha_{2} J_{2} M_{2} \mathbf{p}_{2} m_{s}^{\prime}\right)}{\mathrm{d} \Omega_{2}} \\
& =\sum_{K^{\prime}, K_{2}, K_{\lambda}^{\prime}, K_{s}^{\prime}} \mathcal{A}^{A}\left(K_{1}, K_{2}, K_{\lambda}^{\prime}, K_{s}^{\prime}, K^{\prime}\right) \sum_{N^{\prime}, N_{2}, N_{\lambda}^{\prime}, N_{s}^{\prime}}\left[\begin{array}{l}
K_{\lambda}^{\prime} K_{s}^{\prime} K^{\prime} \\
N_{\lambda}^{\prime} N_{s}^{\prime} N^{\prime}
\end{array}\right]\left[\begin{array}{l}
K_{2} K^{\prime} K_{1} \\
N_{2} N^{\prime} N_{1}
\end{array}\right] \\
& \quad \times T_{N_{2}}^{K_{2}}\left(J_{2}, J_{2}, M_{2} \mid \widehat{J}_{2}\right) T_{N_{s}^{\prime}}^{K_{s}^{\prime}}\left(s, s, m_{s}^{\prime} \mid \widehat{s}\right) \sqrt{4 \pi} Y_{K_{\lambda}^{\prime} N_{\lambda}^{\prime}}\left(\theta_{2}, \phi_{2}\right),
\end{aligned}
$$

where

$$
\begin{aligned}
\mathcal{A}^{A}( & \left.K_{1}, K_{2}, K_{\lambda}^{\prime}, K_{s}^{\prime}, K^{\prime}\right) \\
= & 2 \pi \sum_{\lambda_{1}, j_{1}, \lambda_{2}, j_{2}}\left\langle\alpha_{2} J_{2} \varepsilon_{2} \lambda_{1}\left(j_{1}\right) J_{1}\|H\| \alpha_{1} J_{1}\right\rangle\left\langle\alpha_{2} J_{2} \varepsilon_{2} \lambda_{2}\left(j_{2}\right) J_{1}\|H\| \alpha_{1} J_{1}\right\rangle^{*} \\
& \times\left(2 J_{1}+1\right)\left[\left(2 \lambda_{1}+1\right)\left(2 \lambda_{2}+1\right)\left(2 j_{1}+1\right)\left(2 j_{2}+1\right)\left(2 J_{2}+1\right)(2 s+1)\left(2 K^{\prime}+1\right)\right]^{1 / 2} \\
& \times\left\{\begin{array}{ccc}
J_{2} & j_{1} & J_{1} \\
J_{2} & j_{2} & J_{1} \\
K_{2} & K^{\prime} & K_{1}
\end{array}\right\}\left\{\begin{array}{ccc}
\lambda_{2} & s & j_{2} \\
K_{\lambda} & K_{s}^{\prime} & K^{\prime} \\
\lambda_{1} & s & j_{1}
\end{array}\right\}(-1)^{\lambda_{2}}\left[\begin{array}{ccc}
\lambda_{1} & \lambda_{2} & K_{\lambda}^{\prime} \\
0 & 0 & 0
\end{array}\right] .
\end{aligned}
$$

\section{Dielectronic recombination}

The process of dielectronic recombination (DR) can be written as follows:

$$
A^{+}\left(\alpha_{0} J_{0} M_{0}\right)+\mathrm{e}^{-}\left(\mathbf{p}_{0} m_{0}\right) \rightarrow A^{* *}\left(\alpha_{1} J_{1}\right)\left\{\begin{array}{l}
\rightarrow A\left(\alpha_{2} J_{2} M_{2}\right)+h \nu\left(\epsilon_{q_{2}}, \mathbf{k}_{02}\right), \\
\rightarrow A^{+}\left(\alpha_{3} J_{3} M_{3}\right)+\mathrm{e}^{-}\left(\mathbf{p}_{1} m_{1}\right) .
\end{array}\right.
$$

It is an example of a two-step process. The first step is resonant electron capture. The next step is radiative or Auger decay that were described in Section 4. Two-step approximation for DR may be applied if the interference with the radiative recombination is neglected and the summation over intermediate states $J_{1} M_{1}$, that usually occurs in second-order perturbation theory, is limited to a single resonance. Then, only a summation over the magnetic substates that are not registered is retained. DR process is finished when the photon is emitted. 
In the two-step approximation (see Section 2), the cross-section for DR may be written as

$$
\begin{aligned}
& \frac{\mathrm{d} \sigma\left(\alpha_{0} J_{0} M_{0} \mathbf{p}_{0} m_{0} \rightarrow \alpha_{1} J_{1} \rightarrow \alpha_{2} J_{2} M_{2} \epsilon_{q_{2}} \mathbf{k}_{02}\right)}{\mathrm{d} \Omega} \\
& =2 \pi \sum_{M_{1}, M_{1}^{\prime}}\left\langle\alpha_{2} J_{2} M_{2} \epsilon_{q_{2}} \mathbf{k}_{02}\left|H^{\prime}\right| \alpha_{1} J_{1} M_{1}\right\rangle\left\langle\alpha_{2} J_{2} M_{2} \epsilon_{q_{2}}, \mathbf{k}_{02}\left|H^{\prime}\right| \alpha_{1} J_{1} M_{1}^{\prime}\right\rangle^{*} \\
& \quad \times\left\langle\alpha _ { 1 } J _ { 1 } M _ { 1 } | H ^ { e } | ( \alpha _ { 0 } J _ { 0 } M _ { 0 } \mathbf { p } _ { 0 } m _ { 0 } \rangle \left\langle\alpha_{1} J_{1} M_{1}^{\prime}\left|H^{e}\right|\left(\alpha_{0} J_{0} M_{0} \mathbf{p}_{0} m_{0}\right\rangle^{*}\left[\left(E-E_{1}\right)^{2}+\frac{\Gamma^{2}}{4}\right]^{-1} .\right.\right.
\end{aligned}
$$

Here $H^{\prime}$ and $H^{e}$ is the radiative decay and electrostatic interaction operators, respectively, $\mathrm{d} \Omega$ is the solid angle of the emission of radiation, $E_{1}$ and $E$ is the energy of the intermediate and initial state of the system atom + electron, respectively, and $\Gamma$ denotes the decay width of the intermediate state that includes both radiative and nonradiative decay channels.

In the two-step approximation ( $E \approx E_{1}$ ), the general expression for DR (46) in the case of the interaction of a polarized ion with a polarized electron may be obtained by applying the methods described in $[26,45]$ and is as follows:

$$
\begin{aligned}
& \frac{\mathrm{d} \sigma\left(\alpha_{0} J_{0} M_{0} \mathbf{p}_{0} m_{0} \rightarrow \alpha_{1} J_{1} \rightarrow \alpha_{2} J_{2} M_{2} \epsilon_{q_{2}} \mathbf{k}_{02}\right)}{\mathrm{d} \Omega} \\
& \quad=\frac{2 \pi}{p_{0}^{2}} \sum_{K_{1}, N_{1}} W_{K_{1}, N_{1}}^{\mathrm{c}}\left(\alpha_{0} J_{0} M_{0} \mathbf{p}_{0} m_{0} \rightarrow \alpha_{1} J_{1}\right) \frac{\mathrm{d} \sigma_{K_{1}, N_{1}}^{r}\left(\alpha_{1} J_{1} \rightarrow \alpha_{2} J_{2} M_{2} \epsilon_{q_{2}} \mathbf{k}_{02}\right)}{\mathrm{d} \Omega}\left[\left(E-E_{1}\right)^{2}+\frac{\Gamma^{2}}{4}\right]^{-1} .
\end{aligned}
$$

The resonant electron capture cross-section $W^{\mathrm{c}}$ is inverse to that of Auger decay and is defined by Eq. (43). The expression for the radiative decay probability $\mathrm{d} W^{r} / \mathrm{d} \Omega$ is the same as in Eq. (39).

The DR process is similar to that of resonant electron transfer and excitation (RTE). When highly charged ions interact with low- $Z$ atoms, the differential cross-section for RTE may be determined by using the cross-section of DR and momentum approximation for the distribution of electron charge in the atom [53,54]. Angular distribution of radiation emitted after RTE in collisions of nonpolarized $\mathrm{U}^{90+}$ with a graphite target was investigated in [55]. The process of DR with emission of two and more photons was also treated both in nonrelativistic [56] and fully relativistic [57] approximations.

\section{Practical applications}

General expression for the differential cross-section and probability of the excitation and ionization of polarized atoms and ions by polarized electrons or radiation can be used to obtain much more simple expressions applicable for specific experimental conditions. The authors who used the density matrix formalism to derive the expressions for various parameters describing the polarization usually started from the very beginning by formulating the problem dealing the specific experiment. Below several most important papers are reviewed.

In the case of the photoionization of polarized atoms by polarized radiation, Jacobs [16] obtained the general expression for the differential cross-section, the asymmetry parameters $\beta$ of the angular distribution and $\gamma$, $\delta$, and $\xi$ for the spin polarization of photoelectrons. The statement that the photoion was not detected was assumed from the very beginning. The angular distribution of photoelectrons from nonpolarized atoms was investigated in $[58,59]$. The investigation of the angular distribution of photoelectrons with specific spin orientation in the case of nonpolarized atoms by polarized dipole radiation was carried out by Cherepkov [60]. General expression for the angular distribution and polarization of photoelectrons from nonpolarized atoms in the region of autoionizing states was obtained in [17]. Here the photons of any multipolarity were treated. Later on, the expressions for the cross-sections and asymmetry parameters for the angular distribution of photoelectrons from polarized atoms exposed to polarized radiation in both resonant [20] and nonresonant $[13,18]$ case were obtained. These expressions also found their applications for the investigation of magnetic dichroism in the angular distributions of photoelectrons [61]. Recently, dramatic nondipole effects in 
low-energy photoionization were discovered both theoretically and experimentally [62].

A much larger number of papers is devoted to the investigation of Auger and fluorescence decay of atoms following ionization by electrons and photons. In 1972 and 1974, three papers presented calculations on angular distributions of ionized atom decay products. The polarization of characteristic radiation excited by electron impact [65] and angular distribution of Auger electrons following photoionization $[29,66]$ were calculated not applying the density matrix formalism. Later on, Kabachnik and coworkers [17, 41, 67-71], Lohman et al. [72,73], and Bartschat and Grum-Grzhimailo [74] obtained numerous expressions describing the angular distribution and spin polarization of Auger electrons for various specific experimental conditions. As the expressions were hinted to the applications of noble gases, the initial state of the atom was considered randomly oriented. The atoms could be ionized by polarized dipole photons [67] or nonpolarized electrons $[51,68,72,73]$. Angular distribution of Auger electrons ejected by electron impact from laser-excited and polarized atoms was described in [70]. A resonant cascade model based on a stepwise approach was suggested [41] for the analysis of the angular correlation in the decay of core-excited resonances produced by photoabsorption. The model was applied to the description of angular distributions of Auger electrons and fluorescence.

The alignment of atoms and ions in radiative electron capture (REC) into ground and excited states of ions is recently formed field for the polarization investigation in electron-ion interactions [75-77]. The angular distribution of the decay radiation of recombined ions may provide useful information about the ionic sublevels following electron capture from atoms [78], molecules, and solids. But the parameters of the resonant electron transfer and excitation (RETE) are similar to those of dielectronic recombination. Some special cases of RETE were investigated in [55, 79].

The general expressions presented in Sections 2-5 can be used for the description of the processes mentioned above that were investigated with the help of the density matrix formalism. These general expressions are written in the invariant form of the expansions over the state multipoles, i.e. they are independent of the choice of the coordinate system.

If the atoms or electrons in the initial state are randomly oriented, the general expressions have to be averaged over the projections of the total angular momentum $J$ or electron spin, respectively. When the final states of the products of processes are not detected, the summation over projections over the states of the atom or electron spin should be performed. The integration over the angles of the emission of electrons or radiation should be performed if they are not detected.

The examination of the general expressions (11), (18), (20), (30), (33), (37), (39), and (41) shows that only the tensor $T_{N}^{K}(J, J, M \mid \widehat{J})$ depends on the projection $M$. Summation over $M$ gives [26]

$$
\sum_{M} T_{N}^{K}(J, J, M \mid \widehat{J})=\delta(K, 0) \delta(N, 0) .
$$

Integration over the angles leads to [43]

$\int_{0}^{\pi} \sin \theta \mathrm{d} \theta \int_{0}^{2 \pi} \mathrm{d} \phi Y_{K N}(\theta \phi)=\sqrt{4 \pi} \delta(K, 0) \delta(N, 0)$.

A more complicated case occurs with the polarization of radiation. The nonpolarized radiation usually is represented as a sum of equal parts of the left- and right-polarized radiation since the helicity $q \neq 0$. Thus, for the nonpolarized dipole photon we have

$$
\begin{aligned}
\sum_{q= \pm 1} & T_{N}^{K}\left(1,1, q \mid \widehat{\mathbf{k}}_{0}\right) \\
= & \frac{1}{3} \delta(K, 0) \delta(N, 0) \\
& +\sqrt{\frac{4 \pi}{3}}\left[\begin{array}{ccc}
1 & 1 & 2 \\
1 & -1 & 0
\end{array}\right] Y_{2 N}(\theta, \phi) \delta(K, 2) .
\end{aligned}
$$

This expression can also be used in the case when the polarization of the emitted radiation is not detected.

\subsection{The angular distribution of photoelectrons from unpolarized atoms}

If the initial state of atoms is randomly oriented, the final state of ions and the spin of the photoelectrons are not registered, the summation over the components of the ion state and the spin of the photoelectron, and averaging over the components of the atoms is necessary. From the application of Eq. (48) in (18) it follows that $K_{0}=K_{1}=K_{s}=0, K_{r}=K_{\lambda}=K_{j}=K$. The use of these values in Eq. (18) and the choice of the $z$ axis along the direction of incoming radiation allows us to write the differential cross-section: 


$$
\begin{aligned}
& \frac{\mathrm{d} \sigma\left(\alpha_{0} J_{0} q \rightarrow \alpha_{1} J_{1}\right)}{\mathrm{d} \Omega_{p}} \\
& \quad=\frac{1}{2 J_{0}+1} \sum_{K, k, k^{\prime}} \mathcal{B}^{\mathrm{ph}}\left(0,0, K, K, 0, K, K, k, k^{\prime}\right)(-1)^{k-q}\left[\frac{2 J_{1}+1}{2 k+1}\right]^{1 / 2}\left[\begin{array}{ccc}
k & k^{\prime} & K \\
q-q & 0
\end{array}\right] P_{K}(\cos \theta) .
\end{aligned}
$$

In Eq. (51), $P_{K}(\cos \theta)$ stands for a Legendre polynomial. For the circular polarization of incoming dipole radiation $\left(k=k^{\prime}=q=1\right)$, the cross-section can be written as

$$
\frac{\mathrm{d} \sigma\left(\alpha_{0} J_{0} \rightarrow \alpha_{1} J_{1}\right)}{\mathrm{d} \omega_{p}}=\frac{\sigma\left(J_{0} \rightarrow J_{1}\right)}{4 \pi}\left[1-\frac{1}{2} \beta P_{2}(\cos \theta)\right]
$$

where

$$
\begin{gathered}
\beta=\frac{5 \sqrt{2} \mathcal{B}^{\mathrm{ph}}(0,0,2,2,0,2,2,1,1)}{\mathcal{B}^{\mathrm{ph}}(0,0,0,0,0,0,1,1)}, \\
\sigma\left(\alpha_{0} J_{0} \rightarrow \alpha_{1} J_{1}\right)=\frac{4 \pi}{3\left(2 J_{0}+1\right)} \mathcal{B}^{\mathrm{ph}}(0,0,0,0,0,0,1,1) .
\end{gathered}
$$

The expressions for $\beta$ (53) and $\sigma$ (54) differ from those obtained in [26] and used in the calculations of the angular distribution of $2 p$ photoelectrons from $\mathrm{Na}$ atoms in the ground [80] and excited states [80-82]. The spin polarization parameters $\gamma, \delta$, and $\xi$ were also calculated for the $2 p$ photoionization of $\mathrm{Na}$ in the ground and first excited states [83].

\subsection{Angular distribution of Auger electrons for unpolarized atoms}

The process of the photoionization of an atom $A$ with following Auger decay of a photoion $A^{+}$in a two-step approximation can be written as follows:

$$
\begin{aligned}
A\left(\alpha_{0} J_{0} M_{0}\right)+h \nu\left(\widehat{\epsilon}_{q}, \mathbf{k}_{01}\right) & \rightarrow A^{+}\left(\alpha_{1} J_{1} M_{1}\right)+\mathrm{e}^{-}\left(\mathbf{p}_{1}, m_{s}\right) \\
& \rightarrow A^{2+}\left(\alpha_{2} J_{2} M_{2}\right)+\mathrm{e}^{-}\left(\mathbf{p}_{1}, m_{s}\right)+\mathrm{e}^{-}\left(\mathbf{p}_{2}, m_{s}^{\prime}\right) .
\end{aligned}
$$

In Eq. (55), $\alpha_{0}, \alpha_{1}$, and $\alpha_{2}$ indicate the configuration and other quantum numbers, $J_{0} M_{0}, J_{1} M_{1}$, and $J_{2} M_{2}$ describe the total angular momenta and their magnetic components of the electron cloud of an atom in the initial state, intermediate photoion, and doubly charged ion in the final state, respectively. The photo- and Auger electrons have the momentum $\mathbf{p}_{1}$ and $\mathbf{p}_{2}$, the projection of their spin $s$ is $m_{s}$ and $m_{s}^{\prime}$, respectively. The wave vector of incoming radiation is indicated by $\mathbf{k}_{01}\left(\left|\mathbf{k}_{01}\right|=\omega_{1} / c\right.$, where $\omega_{1}$ is the frequency of radiation).

An expression for the cross-section of the processes (55) was derived by Kupliauskienè and Tutlys [45, 44] and can be written in the form

$$
\begin{aligned}
& \frac{\mathrm{d}^{2} W\left(\alpha_{0} J_{0} M_{0} \widehat{\epsilon}_{q} \mathbf{k}_{01} \rightarrow \alpha_{1} J_{1} \mathbf{p}_{1} m_{s} \rightarrow \alpha_{2} J_{2} M_{2} \mathbf{p}_{2} m_{s}^{\prime}\right)}{\mathrm{d} \Omega_{1} \mathrm{~d} \Omega_{2}} \\
& \quad=\sum_{K_{1}, N_{1}} \frac{\mathrm{d} \sigma_{K_{1} N_{1}}\left(\alpha_{0} J_{0} M_{0} \widehat{\epsilon}_{q} \mathbf{k}_{01} \rightarrow \alpha_{1} J_{1} \mathbf{p} m_{s}\right)}{\mathrm{d} \Omega_{1}} \frac{\mathrm{d} W_{K_{1} N_{1}}\left(\alpha_{1} J_{1} \rightarrow \alpha_{2} J_{2} M_{2} \mathbf{p}_{2} m_{s}^{\prime}\right)}{\mathrm{d} \Omega_{2}} .
\end{aligned}
$$

Here $\mathrm{d} \Omega_{1}$ and $\mathrm{d} \Omega_{2}$ indicate the solid angles of the emission of the photoelectron and Auger electron, respectively.

The probability describing the angular distribution of Auger electrons from unpolarized atoms simplifies as a result of the summation of (56) over the magnetic components of the spins of photo- and Auger electrons, the total 
angular momentum of a doubly charged ion, integration over the angles of photoelectron, and averaging over the magnetic components of an atom. In the case of dipole approximation, it acquires the following form:

$$
\begin{aligned}
\frac{\mathrm{d} W\left(J_{0} \rightarrow J_{1} \rightarrow J_{2} \mathbf{p}_{2}\right)}{\mathrm{d} \Omega_{2}} & =\sum_{K_{1}} \mathcal{A}^{A}\left(K_{1}, 0, K_{1}, 0, K_{1}\right) B\left(K_{1}\right) P_{K_{1}}\left(\cos \theta_{2}\right) \\
& =\mathcal{A}^{A}(0,0,0,0,0) B(0)\left[1+\beta P_{2}\left(\cos \theta_{2}\right)\right] .
\end{aligned}
$$

Here

$$
\begin{aligned}
B\left(K_{1}\right) & =\frac{4 \pi}{2 J_{0}+1}\left[\frac{2 K_{1}+1}{3}\right]^{1 / 2}\left[\begin{array}{ccc}
1 & 1 & K_{1} \\
1-1 & 0
\end{array}\right] \mathcal{B}\left(K_{1}, 0, K_{1}, 0,0,0, K_{1}, 1,1\right), \\
\beta & =\frac{\mathcal{A}^{A}(2,0,2,0,2) B(2)}{\mathcal{A}^{A}(0,0,0,0,0) B(0)}=\frac{\mathcal{A}^{A}(2,0,2,0,2)}{\mathcal{A}^{A}(0,0,0,0,0)} A_{2},
\end{aligned}
$$

where $A_{2}$ is the alignment [26]. Its expression coincides with that presented by Kabachnik and Sazhina [69] if the expressions for $B\left(K_{1}\right)$ would be inserted.

In the case of unpolarized atoms, the expression for the probability describing angular correlations between photo- and Auger electrons is obtained by summation of Eq. (56) over magnetic components of the spins of photo- and Auger electrons, the angular momentum of a doubly charged ion and averaging over magnetic components of an atom. For circularly polarized dipole ionizing radiation, it has the following expression:

$$
\begin{aligned}
\frac{\mathrm{d}^{2} W\left(J_{0} \rightarrow J_{1} \mathbf{p}_{1} \rightarrow J_{2} \mathbf{p}_{2}\right)}{\mathrm{d} \Omega_{1} \mathrm{~d} \Omega_{2}}= & \sum_{K_{1} N_{1}} \mathcal{A}\left(K_{1}, 0, K_{1}, 0, K_{1}\right)\left[\frac{4 \pi}{2 K_{1}+1}\right]^{1 / 2} Y_{K_{1} N_{1}}\left(\theta_{2}, \phi_{2}\right) \sum_{K_{r}, K_{\lambda}} B^{\prime}\left(K_{1}, K_{\lambda}, K_{r}\right) \\
& \times \sum_{N_{r}, N_{\lambda}}\left[\begin{array}{l}
K_{1} K_{\lambda} K_{r} \\
N_{1} N_{\lambda} N_{r}
\end{array}\right] \frac{4 \pi}{\sqrt{2 K_{r}+1}} Y_{K_{r} N_{r}}^{*}\left(\theta_{0}, \phi_{0}\right) Y_{K_{\lambda} N_{\lambda}}\left(\theta_{1}, \phi_{1}\right) .
\end{aligned}
$$

Here

$$
\begin{aligned}
B\left(K_{1}, K_{\lambda}, K_{r}\right)= & \frac{1}{2 J_{0}+1}\left[\frac{\left(2 K_{1}+1\right)\left(2 K_{r}+1\right)}{3}\right]^{1 / 2}\left[\frac{2 K_{1}+1}{3}\right]^{1 / 2}\left[\begin{array}{ccc}
1 & 1 & K_{r} \\
1 & -1 & 0
\end{array}\right] \\
& \times \mathcal{B}\left(K_{1}, 0, K_{r}, K_{\lambda}, 0, K_{\lambda}, K_{1}, 1,1\right) .
\end{aligned}
$$

More examples of the practical application of the general expressions can be found in $[26,42,44-46,52]$.

\section{Concluding remarks}

A method for the derivation of general expressions for the cross-section and transition probability describing the polarization states of all particles participating in the interaction of polarized photons and electrons with polarized atoms is developed. It is alternative to the density matrix formalism. The graphical technique of the angular momentum applied for the integration over angular and spin variables of the matrix elements as well as for the expansion of the crosssections and probabilities over the spherical tensors enables us to obtain the most general expressions for the cross-sections. Irreducible tensors are selected for the description of polarization because they have the sim- plest possible behaviour under changes of directions. The method is also generalized for the multistep processes.

The following processes playing very important role in plasma are investigated:

- excitation and ionization of atoms by photons;

- excitation and ionization of atoms by electron impact;

- radiative recombination of an ion and an electron;

- radiative and Auger decay of excited and ionized atoms.

The dielectronic recombination of an ion with an electron is an example of a two-step process. 
The practical application of the general expressions for the description of more simple processes under specific experimental conditions is easy to accomplish. The asymmetry parameter of the angular distribution of photoelectrons and Auger electrons following photoionization of unpolarized atoms as well as the parameters describing the angular correlations between the photo- and Auger electrons are obtained. These examples demonstrate the way for the derivation of more simple expressions. All expressions are presented in a similar form convenient for development of computer software and practical applications.

\section{References}

[1] A.P. Jucys and A.A. Bandzaitis, Theory of Angular Momentum in Quantum Mechanics (Mintis, Vilnius, 1965) [in Russian].

[2] A.P. Jucys and A.J. Savukynas, Mathematical Foundations of the Atomic Theory (Mintis, Vilnius, 1972) [in Russian].

[3] J.H. Macek, Alignment and orientation: Opening remarks, in: Atomic Physics, Vol. 16, eds. W.E. Baylis and G.W.F. Drake (American Institute of Physics, New York, 1999) pp. 234-236.

[4] U. Heinzmann, Experimental determination of the phase difference of continuum wavefunctions describing the photoionization process of xenon atoms, II. Evaluation of the matrix elements and their phase differences and their comparison with data in the discrete spectral range in application of the multichannel quantum defect theory, J. Phys. B 13, 4367-4381 (1980).

[5] S.A. Kazantsev and J.-C. Henoux, Polarization Spectroscopy of Ionized Gases (Kluwer, Dordrecht/Boston/ London, 1995).

[6] A.M. Urnov, Historical overview of plasma polarization spectroscopy, in: Proceedings of the JapanUS Workshop on Plasma Polarization Spectroscopy and the International Seminar on Plasma Polarization Spectroscopy (Research International Center, Nagoya, 1998) pp. 1-8.

[7] J.C. Kieffer, J.P. Matte, H. Pèpin, M. Chaker, Y. Beoudain, T.W. Johnston, C.H. Chien, S. Coe, G. Moorou, and J. Dubou, Electron distribution anisotropy in laserproduced plasmas from X-ray line polarization measurements, Phys. Rev. Lett. 68, 480-483 (1992).

[8] T. Fujimoto, H. Sahara, T. Kawachi, T. Kallstenius, M. Goto, H. Kawase, T. Furukubo, T. Mackawa, and Y. Terumichi, Polarization of impurity emission lines from tokamak plasma, Phys. Rev. E 54, R2240-R2243 (1996).
[9] V.A. Veretennikov, A.E. Gurei, A.N. Dolgov, V.V. Korneev, and O.G. Semenov, The polarization of line $\mathrm{X}$-ray radiation from impulse discharge plasma, Pis'ma Zh. Eksp. Teor. Fiz. 47, 29-31 (1988).

[10] S.A. Kazantsev, The application of the self-alignment for the astrophysical and laboratory plasma, Uspekhi Fiz. Nauk 139, 621-666 (1983).

[11] A. von dem Borne, T. Dohrmann, A. Verweyen, and B. Sonntag, Dichroism in the $3 p$ photoionization of polarized Cr atoms, Phys. Rev. Lett. 78, 4019-4022 (1997).

[12] G. Prümper, O. Geßner, B. Zimmermann, J. Viefhaus, R. Hentger, H. Kleinpoppen, and U. Becker, Absorption of circularly polarized VUV radiation in polarized iron vapor, J. Phys. B 34, 2707-2714 (2001).

[13] N.A. Cherepkov, V.V. Kuznetsov, and V.A. Verbitskii, Photoionization of polarized atoms, J. Phys. B 28, 1221-1239 (1995).

[14] U. Fano, Description of states in quantum mechanics by density matrix and operator technique, Rev. Mod. Phys. 29, 74-93 (1957).

[15] U. Fano and J.H. Macek, Impact excitation and polarization of the emitted light, Rev. Mod. Phys. 45, 553573 (1973).

[16] V.L. Jacobs, Theory of atomic polarization measurements, J. Phys. B 5, 2257-2271 (1972).

[17] N.M. Kabachnik and I.P. Sazhina, Angular distribution and polarization of photoelectrons in the region of resonances, J. Phys. B 9, 1681-1697 (1976).

[18] H. Klar, Polarization of fluorescence radiation following atomic photoionization, J. Phys. B 13, 2037-2049 (1980).

[19] H. Klar and H. Kleinpoppen, Angular distribution of photoelectrons from polarized atoms exposed to polarized radiation, J. Phys. B 15, 933-950 (1982).

[20] S. Baier, A.N. Grum-Grzhimailo, and N.M. Kabachnik, Angular distribution of photoelectrons in resonant photoionization of polarized atoms, J. Phys. B 27, 3363-3388 (1994).

[21] A.N. Grum-Grzhimailo, K. Bartschat, N. Feuerstein, and W. Mehlhorn, Near threshold structure in electroncollision-induced alignment of excited atomic states, Phys. Rev. A 60, R1751-R1754 (1994).

[22] A.N. Grum-Grzhimailo and N.M. Kabachnik, Linear magnetic dichroism in fluorescence spectra, Phys. Lett. A 264, 192-197 (1999).

[23] V.V. Balashov, A.N. Grum-Grzhimailo, and N.M. Kabachnik, Polarization and Correlation Phenomena in Atomic Collisions. A Practical Theory Course (Kluwer, New York, 2000).

[24] K. Blum, Density Matrix Theory and Applications, 2nd edn. (Plenum, New York, 1996). 
[25] A. Kupliauskienè, N. Rakštikas, and V. Tutlys, General expression of the photoionization cross-section of an atom in polarized $L S$ state, Lithuanian J. Phys. 40, 311-320 (2000).

[26] A. Kupliauskienè, N. Rakštikas, and V. Tutlys, Polarization studies in the photoionization of atoms using a graphical technique, J. Phys. B 34, 1783-1803 (2001).

[27] Z. Rudzikas, Theoretical Atomic Spectroscopy (ManyElectron Atoms) (Cambridge University Press, Cambridge, 1995).

[28] U. Fano and D. Dill, Angular momentum transfer in the theory of angular distributions, Phys. Rev. A 6, 185-192 (1972).

[29] B. Cleff and W. Mehlhorn, On the angular distribution of Auger electrons following impact ionization, J. Phys. B 7, 593-604 (1974).

[30] I.B. Levinson, Sums of the products of Wigner coefficients and their graphical representation, Proc. Inst. Phys. Techn. 2, 17-29 (1956) [in Russian].

[31] D.M. Brink and G.R. Satchler, Angular Momentum (Oxford University Press, Oxford, 1968).

[32] J.S. Briggs, Evaluation of matrix elements from a graphical representation of the angular integrals, Rev. Mod. Phys. 43, 189-230 (1971).

[33] E. El-Baz and B. Castel, Graphical Methods of Spin Algebras in Atomic, Nuclear and Particle Physics (Marcel Dekker, Oxford, 1972).

[34] K.-N. Huang, Graphical evaluation of relativistic matrix elements, Rev. Mod. Phys. 51, 215-236 (1979).

[35] G. Merkelis, Graphical method of evaluation of matrix elements in the second quantization representation, Physica Scripta 63, 289-305 (2001).

[36] Ph. Golecki and H. Klar, (e, 2e) from laser-excited atoms with spin-polarized electrons, J. Phys. B 32, 1647-1656 (1999).

[37] H. Aksela, Resonant Auger spectroscopy of atoms and molecules, J. Electr. Spectr. Relat. Phenomena 72, 235-242 (1995).

[38] K. Ueda, Y. Shimizu, H. Chiba, Y. Sato, M. Kitajima, H. Tanaka, and N.M. Kabachnik, Experimental determination of Auger-decay amplitudes from the angular correlations in Auger cascades following the $2 p \rightarrow 4 s$ photoexcitation of Ar, Phys. Rev. Lett. 83, 5463-5466 (1999).

[39] B. Langer, N. Berrah, A. Farhat, M. Humphrey, D. Cubaynes, A. Menzel, and U. Becker, Angular distributions of resonant and non-resonant Auger electrons as a test case for the validity of spectator model: The $\operatorname{argon} L_{2} M M$ case, J. Phys. B 30, 4255-4266 (1977).

[40] P. O'Keeffe, S. Aloise, M. Meyer, and A.N. GrumGrzhimailo, Circular polarization of ion fluorescence completing the analysis of resonant $\mathrm{Xe}^{*} 4 d_{5 / 2}^{-1} 6 p$ Auger decay, Phys. Rev. Lett. 90, 023002(4) (2003).
[41] N.M. Kabachnik, I.P. Sazhina, and K. Ueda, Angular distribution of Auger electrons and fluorescence in cascades and resonantly enhanced transitions, J. Phys. B 32, 1769-1781 (1999).

[42] A. Kupliauskienè, Photoexcitation of polarized atoms by polarized radiation, Lithuanian J. Phys. 44(1), 17-26 (2004).

[43] D.A. Varshalovich, A.N. Moskalev, and V.K. Khersonskii, Quantum Theory of Angular Momentum (World Scientific, Singapore, 1988).

[44] A. Kupliauskienè and V. Tutlys, Application of graphical technique for Auger decay following photoionization of atoms, Physica Scripta 67, 290-300 (2003).

[45] A. Kupliauskienė and V. Tutlys, Auger decay probability following photoionization of atoms, Lithuanian J. Phys. 43, 27-34 (2003).

[46] A. Kupliauskienė and K. Glemža, General expression for ionization cross-section of polarized atoms by polarized electrons, Lithuanian J. Phys. 43, 89-97 (2003).

[47] P. Serapinas and A. Kupliauskienè, On current filament formation in arc cathode plasma, J. Phys. D 27, 330337 (1994).

[48] A. Dorn, A. Elliott, J. Lower, E. Weigold, J. Berakdar, A. Engelns, and H. Klar, Orientational dichroism in the electron-impact ionization of laser-oriented atomic sodium, Phys. Rev. Lett. 80, 257-260 (1998).

[49] J. Lower, E. Weigold, J. Berakdar, and S. Mazevet, Magnetic and orbital dichroism in (e, 2e) ionization of sodium, Phys. Rev. Lett. 86, 624-627 (2001).

[50] S. Gelfort, H. Kerkow, P.V. Petukhov, and E.A. Romanovskii, Influence of Coster-Kroning transitions on the polarization of $L$-shell X-rays induced by proton impact, Zh. Eksp. Teor. Fiz. 113, 2005-2010 (1998) [in Russian].

[51] E.G. Berezhko and N.M. Kabachnik, Theoretical study of inner-shell alignment of atoms in electron impact ionization: Angular distribution and polarization of X-rays and Auger electrons, J. Phys. B 10, 2467-2477 (1977).

[52] A. Kupliauskienė and V. Tutlys, Angular distribution and polarization of radiation following photoionization of polarized atoms, Physica Scripta (accepted).

[53] Y. Hann and K.J. LaGattuta, Dielectronic recombination and related resonance processes, Physics Reports (Review Section of Physics Letters) 166, 195268 (1988).

[54] A.V. Kupliauskiene and R.L. Furmonavichyute, Crosssections for resonance charge exchange with electron excitation due to collisions of $\mathrm{Ca}^{17+}$ with $\mathrm{He}$ and $\mathrm{H}_{2}$, Opt. Spektrosk. (USSR) 71, 13-15 (1991).

[55] M. Gail, N. Grün, and W. Scheid, Angular distribution of radiation emitted after resonant transfer and excitation, J. Phys. B 31, 4645-4654 (1998). 
[56] V.V. Balashov, I.V. Bondarenko, V.K. Dolinov, and S.I. Strachova, Angular anisotropy of the cascade photons in the dielectronic recombination of ions, Opt. Spektrosk. 77, 801-806 (1994).

[57] S. Zakowicz, W. Scheid and N. Grün, Dielectronic recombination into hydrogen-like heavy ions with emission of two photons, J. Phys. B 37, 131-145 (2004).

[58] J. Cooper and R.N. Zare, Angular distribution of photoelectrons, J. Chem. Phys. 48, 942-943 (1968).

[59] D. Dill, A.F. Starace, and S.T. Manson, Effects of anisotropic electron-ion interactions in atomic photoelectron angular distributions, Phys. Rev. A 11, 15961606 (1975).

[60] N.A. Cherepkov, Angular distribution of photoelectrons with specific spin orientation, Zh. Eksp. Teor. Fiz. 65, 933-946 (1973) [in Russian].

[61] O. Plotzke, G. Prümper, B. Zimmermann, U. Becker, and H. Kleinpoppen, Magnetic dichroism in the angular distribution of atomic oxygen $2 p$ photoelectrons, Phys. Rev. Lett. 77, 2642-2645 (1996).

[62] O. Hemmers et al., Dramatic nondipole effects in lowenergy photoionization: Experiment and theoretical study of Xe 5s, Phys. Rev. Lett. 91, 053002 (2003).

[63] C. Pan and A.F. Starace, Angular distributions for nearthreshold (e, 2e) processes for Li and Mg, Phys. Rev. A 47, 2389-2392 (1993).

[64] M. Streun et al., Spin dependence of $(e, 2 e)$ collisions on lithium at 54.4 eV, J. Phys. B 31, 4401-4411 (1998).

[65] S.C. McFarlane, The polarization of characteristic X-radiation excited by electron impact, J. Phys. B 5, 1906-1915 (1972).

[66] S. Flügge, W. Mehlhorn, and V. Schmidt, Angular distribution of Auger electrons following photoionization, Phys. Rev. Lett. 29, 7-9 (1972).

[67] E.G. Berezhko, N.M. Kabachnik, and V.S.Rostovsky, Potential-barrier effects in inner-shell photoionization and their influence on the anisotropy of X-rays and Auger electrons, J. Phys. B 11, 1749-1758 (1978).

[68] E.G. Berezhko, N.M. Kabachnik, and V.V. Sizov, The theory of coincidence experiments on electron impact ionization of inner atomic shells, J. Phys. B 11, 18191832 (1978).

[69] N.M. Kabachnik and I.P. Sazhina, Angular distribution and spin polarization of Auger electrons, J. Phys. B 17, 1335-1342 (1984).

[70] V.V. Balashov, A.N. Grum-Grzhimailo, and N.M. Kabachnik, Angular distribution of autoionization and Auger electrons ejected by electron impact from laserexcited and polarized atoms, J. Phys. B 30, 1269-1291 (1997).
[71] K. Ueda, Y. Shimizu, H. Chiba, M. Kitajima, H. Tanaka, S. Fritzsche, and N.M. Kabachnik, Experimental and theoretical study of the Auger cascade following $2 p \rightarrow 4 s$ photoexcitation in Ar, J. Phys. B 34, 107119 (2001).

[72] K. Blum, B. Lohmann, and E. Taute, Angular distribution and polarization of Auger electrons, J. Phys. B 19, 3815-3825 (1986).

[73] U. Kleiman and B. Lohmann, Large dynamic spin polarization parameters for diagram $L_{3} M_{1} M_{4,5}$ Auger transitions, J. Phys. B 33, 2653-2663 (2000).

[74] K. Bartschat and A.N. Grum-Grzhimailo, Vector $\left(\mathrm{e}, \mathrm{e}^{\prime} \gamma\right)$ correlations in ionization-excitation of $\mathrm{He}$ by electron impact, J. Phys. B 35, 5035-5050 (2002).

[75] J. Eichler, A. Ichihara, and T. Shirai, Alignment caused by photoionization and in radiative electron capture into excited states of hydrogenic high- $Z$ ions, Phys. Rev. A 58, 2128-2135 (1998).

[76] J. Eichler and A. Ichihara, Polarization of photons emitted in radiative electron capture by bare high- $Z$ ions, Phys. Rev. A 65, 052716 (2002).

[77] A. Surzhykov, S. Fritzsche, and Th. Stöhlker, Twostep radiative recombination of polarized electrons into bare, high- $Z$ ions, Nucl. Instrum. Methods Phys. Res. B 205, 391-394 (2003).

[78] P.D. Fainstein, L. Gulyas, F. Martin, and A. Salin, Angular asymmetry of low-energy electron emission in ion-atom collisions, Phys. Rev. A 53, 3243-3246 (1996).

[79] H. Tanuma, T. Hayakawa, C. Verzani, H. Kano, H. Watanabe, B.D. DePaola, and N. Kobayashi, Polarization spectroscopy of $\mathrm{O}^{5+}\left(1 s^{2} 3 p\right)$ states produced in the collisions of $\mathrm{O}^{6+}$ with $\mathrm{He}$ and $\mathrm{H}_{2}$, J. Phys. B 33, 5091-5098 (2000).

[80] A. Kupliauskienè, On the application of relaxedorbital and sudden perturbation approximations for the photoionization of atoms, J. Phys. B 34, 345-361 (2001).

[81] N. Rakštikas and A. Kupliauskienè, Strong dependence of the $2 p$ photoionization cross sections of $\mathrm{Na}$ atoms on valence electron state, Physica Scripta 58, 587-594 (1998).

[82] A. Kupliauskienė and J. Lipinskaja, The peculiarities of the angular distribution of photoelectrons from the $2 p$ shell of excited Na, Lithuanian J. Phys. 41, 208-212 (2001).

[83] D. Jurčiukonis and A. Kupliauskienè, Investigation of the influence of valence electron excitation on the polarization of photoelectrons from $\mathrm{Na}$ atoms, Lithuanian J. Phys. 41, 242-246 (2001). 


\title{
ATOMO TEORIJOS METODAI ATOMŲ SAૃVEIKAI SU FOTONAIS IR ELEKTRONAIS TIRTI
}

\author{
A. Kupliauskienè
}

VU Teorinès fizikos ir astronomijos institutas, Vilnius, Lietuva

\section{Santrauka}

Sklaidos uždaviniuose sklaidomosios dalelès kryptis yra apibrěžta, todèl reakcijos produktų būsenų užpildai būdinga asimetrija šios krypties atžvilgiu. Iki šiol tokia poliarizacija ir asimetrija buvo tiriamos tankio matricos metodais. Prieš ketvertą metu poliarizacijos reiškiniams, kai atomai ir jonai sąveikauja su elektronais ir fotonais, nagrinèti buvo pritaikyti atomo teorijos metodai, kurie iki šiol buvo taikomi izoliuotiems atomams tirti, neatsižvelgiant $i$ išskirtą krypti erdvèje. Apžvelgti darbai, skirti poliarizuotų atomų sąveikos su poliarizuotais fotonais ir elektronais skerspjūviu bendriausioms išraiškoms nereliatyvistiniu artèjimu surasti. Judejjimo kiekio momento grafinè technika pritaikyta šuolio operatoriu matriciniu elementu kvadratams integruoti kampiniu ir sumuoti suki- ninių kintamųjų atžvilgiu. Skerspjūvių išraiškos užrašytos daugialypiais sferinių multipolių skleidiniais, kadangi sferiniai tenzoriai transformuojasi paprasčiausiai, keičiant matavimo kryptis. Pateiktas būdas fotonų ar elektronų spinduliuotei po atomo ar jono jonizacijos, sužadinimo ar rekombinacijos dvipakopiu artèjimu nagrinèti.

Išnagrinèti svarbiausi plazmoje vykstantys vyksmai: atomų ir jonų sužadinimas ir jonizacija fotonais bei elektronais, jono ir elektrono fotorekombinacija, spinduliavimo ir Auger šuoliai sužadintuose ir jonizuotuose atomuose. Dvipakopi vyksmą iliustruoja dvielektronè rekombinacija. Taip pat parodyta, kaip galima surasti skerspjūvių išraiškas konkretiems eksperimentams aprašyti. Visų išraiškų forma paprasta, patogi kompiuterinėms programoms rašyti. 